TRANSACTIONS OF THE

AMERICAN MATHEMATICAL SOCIETY

Volume 358, Number 6, Pages 2425-2446

S 0002-9947(06)04081-5

Article electronically published on January 24, 2006

\title{
ON THE SHAPE OF THE MODULI OF SPHERICAL MINIMAL IMMERSIONS
}

\author{
GABOR TOTH
}

\begin{abstract}
The DoCarmo-Wallach moduli space parametrizing spherical minimal immersions of a Riemannian manifold $M$ is a compact convex body in a linear space of tracefree symmetric endomorphisms of an eigenspace of $M$. In this paper we define and study a sequence of metric invariants $\sigma_{m}, m \geq 1$, associated to a compact convex body $\mathcal{L}$ with base point $\mathcal{O}$ in the interior of $\mathcal{L}$. The invariant $\sigma_{m}$ measures how lopsided $\mathcal{L}$ is in dimension $m$ with respect to $\mathcal{O}$. The results are then appplied to the DoCarmo-Wallach moduli space. We also give an efficient algorithm to calculate $\sigma_{m}$ for convex polytopes.
\end{abstract}

\section{INTRODUCTION}

Let $\mathcal{L}$ be a compact convex body in a Euclidean vector space $\mathcal{E}$ and $\mathcal{O} \in \operatorname{int} \mathcal{L}$, a base point in the interior of $\mathcal{L}$. Given $C \in \partial \mathcal{L}$, the line passing through $\mathcal{O}$ and $C$ intersects $\partial \mathcal{L}$ in another point. We call this the opposite of $C$ with respect to $\mathcal{O}$ and denote it by $C^{o}$. Clearly, $\left(C^{o}\right)^{o}=C$.

The distortion function $\Lambda: \partial \mathcal{L} \rightarrow \mathbf{R}$ is defined by

$$
\Lambda(C)=\frac{d(C, \mathcal{O})}{d\left(C^{o}, \mathcal{O}\right)}, \quad C \in \partial \mathcal{L},
$$

where $d\left(X, X^{\prime}\right)=\left|X-X^{\prime}\right|$ is the Euclidean distance.

The distortion measures how far $\mathcal{L}$ is from being (centrally) symmetric with respect to $\mathcal{O}$. Clearly, $\Lambda\left(C^{o}\right)=1 / \Lambda(C)$. The distortion function $\Lambda$ is continuous, since both the numerator and the denominator in (1) are continuous in $C \in \partial \mathcal{L}$ [1, 2]. In addition, we have

$$
\frac{1}{\operatorname{dim} \mathcal{E}} \leq \Lambda \leq \operatorname{dim} \mathcal{E},
$$

provided that $\mathcal{O} \in \mathcal{L}$ is chosen appropriately [1].

Let $m \geq 1$. A finite set $\left\{C_{0}, \ldots, C_{m}\right\}$ is called an $m$-configuration (relative to $\mathcal{O})$ if $\left\{C_{0}, \ldots, C_{m}\right\} \subset \partial \mathcal{L}$ and $\mathcal{O}$ is contained in the convex hull $\left[C_{0}, \ldots, C_{m}\right]$. (Note that $\left[C_{0}, \ldots, C_{m}\right]$ is a convex polytope in its affine span and its dimension is maximal $(=m)$ iff it is an $m$-simplex.)

Let $\mathcal{C}_{m}(\mathcal{L})$ denote the set of all $m$-configurations of $\mathcal{L}$. We define

$$
\sigma_{m}(\mathcal{L})=\inf _{\left\{C_{0}, \ldots, C_{m}\right\} \in \mathcal{C}_{m}(\mathcal{L})} \sum_{i=0}^{m} \frac{1}{1+\Lambda\left(C_{i}\right)} .
$$

Received by the editors April 7, 2004.

2000 Mathematics Subject Classification. Primary 53C42.

Key words and phrases. Convex set, extremal point, distortion.

(C)2006 American Mathematical Society 
An $m$-configuration $\left\{C_{0}, \ldots, C_{m}\right\}$ is called minimal if

$$
\sigma_{m}(\mathcal{L})=\sum_{i=0}^{m} \frac{1}{1+\Lambda\left(C_{i}\right)}
$$

Minimal configurations always exist since $\mathcal{L}$ is compact.

Since a 1-configuration of $\mathcal{L}$ is an opposite pair of points $\left\{C, C^{\circ}\right\} \subset \partial \mathcal{L}$ and $\Lambda\left(C^{o}\right)=1 / \Lambda(C)$, we have

$$
\frac{1}{1+\Lambda(C)}+\frac{1}{1+\Lambda\left(C^{o}\right)}=1
$$

This gives $\sigma_{1}(\mathcal{L})=1$

We have the obvious upper estimate

$$
\sigma_{m+k}(\mathcal{L}) \leq \sigma_{m}(\mathcal{L})+\frac{k}{1+\max _{\partial \mathcal{L}} \Lambda}, m \geq 1, k \geq 0
$$

In particular, the difference $\sigma_{m+1}(\mathcal{L})-\sigma_{m}(\mathcal{L})$ is at most $1 /\left(1+\max _{\partial \mathcal{L}} \Lambda\right)$.

The most important invariant is $\sigma(\mathcal{L})=\sigma_{n}(\mathcal{L})$, where $n=\operatorname{dim} \mathcal{L}=\operatorname{dim} \mathcal{E}$. As shown in [4, for $k \geq 1$, we have

$$
\sigma_{n+k}(\mathcal{L})=\sigma(\mathcal{L})+\frac{k}{1+\max _{\partial \mathcal{L}} \Lambda}
$$

Equivalently, the sequence $\left\{\sigma_{m}(\mathcal{L})\right\}_{m \geq n}$ is arithmetic with difference $1 /\left(1+\max _{\partial \mathcal{L}} \Lambda\right)$. Clearly, for $m \leq n$, we have

$$
\sigma_{m}(\mathcal{L})=\inf _{\mathcal{O} \in \mathcal{F} \subset \mathcal{E}, \operatorname{dim} \mathcal{F}=m} \sigma(\mathcal{L} \cap \mathcal{F}),
$$

where the infimum is over affine subspaces $\mathcal{F} \subset \mathcal{E}$. (In the infimum, $\sigma(\mathcal{L} \cap \mathcal{F})=$ $\sigma_{m}(\mathcal{L} \cap \mathcal{F})$, since $\operatorname{dim}(\mathcal{L} \cap \mathcal{F})=\operatorname{dim} \mathcal{F}=m$.)

In 4 the following result was proved.

Theorem A. Let $\mathcal{L} \subset \mathcal{E}$ be a compact convex body in a Euclidean vector space $\mathcal{E}$ of dimension $n$ with base point $\mathcal{O}$. Let $m \geq 1$. Then

$$
1 \leq \sigma_{m}(\mathcal{L}) \leq \frac{m+1}{2} .
$$

If $\sigma_{m}(\mathcal{L})=1$, then $m \leq n$ and there exists an affine subspace $\mathcal{F} \subset \mathcal{E}, \mathcal{O} \in \mathcal{F}$, of dimension $m$ such that $\mathcal{L} \cap \mathcal{F}$ is an m-simplex. In fact, in this case a minimal configuration $\left\{C_{0}, \ldots, C_{m}\right\} \in \mathcal{C}(\mathcal{L} \cap \mathcal{F})$ is unique and is given by the set of vertices of $\mathcal{L} \cap \mathcal{F}$. Moreover, minimality

$$
\sum_{i=0}^{m} \frac{1}{1+\Lambda\left(C_{i}\right)}=1
$$

implies

$$
\sum_{i=0}^{m} \frac{1}{1+\Lambda\left(C_{i}\right)} C_{i}=0
$$

Conversely, if $\mathcal{L}$ has a simplicial intersection with an $m$-dimensional affine subspace $\mathcal{F} \ni \mathcal{O}$, then $\sigma_{m}(\mathcal{L})=1$.

For $m \geq 2, \sigma_{m}(\mathcal{L})=(m+1) / 2$ iff $\Lambda=1$ on $\partial \mathcal{L}$, that is, iff $\mathcal{L}$ is symmetric.

In general, the sequence $\left\{\sigma_{m}(\mathcal{L})\right\}_{m=1}^{n}$ is not arithmetic but superadditive in the following sense. 
Theorem B. We have

$$
\sigma_{m+k}(\mathcal{L}) \geq \sigma_{m+1}(\mathcal{L})+\sigma_{k}(\mathcal{L})-1, m \geq 0, k \geq 1 .
$$

We can write $(8)$ in the more symmetric form

$$
\sigma_{m+k}(\mathcal{L})-\sigma_{m+1}(\mathcal{L}) \geq \sigma_{k}(\mathcal{L})-\sigma_{1}(\mathcal{L})
$$

Setting $k=2$ and using $\sigma_{2}(\mathcal{L}) \geq 1$ (Theorem A), we immediately obtain that the sequence $\left\{\sigma_{m}(\mathcal{L})\right\}_{m=1}^{\infty}$ is increasing. Since $\sigma_{1}(\mathcal{L})=1$, there is a largest integer $r(\mathcal{L}) \geq 1$ such that $\sigma_{m}(\mathcal{L})=1$ for $1 \leq m \leq r(\mathcal{L})$. By Theorem $\mathrm{A}, r(\mathcal{L})$ is the maximum dimension of an affine subspace $\mathcal{F}(\ni \mathcal{O})$ of $\mathcal{E}$ that intersects $\mathcal{L}$ in a simplex. The obvious lower estimate

now gives

$$
\frac{k+1}{1+\max _{\partial \mathcal{L}} \Lambda} \leq \sigma_{k}(\mathcal{L})
$$

$$
r(\mathcal{L}) \leq\left[\max _{\partial \mathcal{L}} \Lambda\right]
$$

We now turn to applications to moduli spaces. Let $\mathcal{H}$ be a Euclidean vector space, and let $S_{0}^{2}(\mathcal{H})$ denote the space of symmetric endomorphisms of $\mathcal{H}$ with vanishing trace. The reduced moduli space

$$
\mathcal{K}_{0}=\mathcal{K}_{0}(\mathcal{H})=\left\{C \in S_{0}^{2}(\mathcal{H}) \mid C+I \geq 0\right\}
$$

is a compact convex body in $S_{0}^{2}(\mathcal{H})$ with centroid $\mathcal{O}$ at the origin.

All moduli spaces considered here are the intersections of $\mathcal{K}_{0}$ with linear subspaces $\mathcal{E} \subset S_{0}^{2}(\mathcal{H})$. More specifically, the fundamental problem in the study of the DoCarmo-Wallach moduli spaces is to describe the shape of the compact convex body that parametrizes spherical eigenmaps and minimal immersions $f: M \rightarrow S_{V}$ of a compact (homogeneous) Riemannian manifold $M$ into the unit sphere $S_{V}$ of a Euclidean vector space $V$, for various $V$. The components of such maps are in an eigenspace $\mathcal{H}=\mathcal{H}_{\lambda}$ corresponding to an eigenvalue $\lambda$ of the Laplace-Beltrami operator acting on functions of $M$. To indicate the dependence of these maps on $\lambda$, we will call them $\lambda$-eigenmaps; for minimal immersions the induced metric on $M$ is $\lambda / \operatorname{dim} M$-times the original metric on $M$. The moduli spaces are $\mathcal{L}_{\lambda}=\mathcal{K}_{0} \cap \mathcal{E}_{\lambda}$ (for $\lambda$-eigenmaps) and $\mathcal{M}_{\lambda}=\mathcal{K}_{0} \cap \mathcal{F}_{\lambda}$ (for spherical minimal immersions), where $\mathcal{F}_{\lambda} \subset \mathcal{E}_{\lambda}$, and $\mathcal{E}_{\lambda}$ (and consequently, $\mathcal{F}_{\lambda}$ ) are orthogonal to certain rank one endomorphisms of $\mathcal{H}_{\lambda}$ defined by the Dirac delta map $\delta: M \rightarrow \mathcal{H}_{\lambda}^{*}[5$.

In view of Theorem $\mathrm{A}$, the invariants $\sigma_{m}\left(\mathcal{L}_{\lambda}\right)$ and $\sigma_{m}\left(\mathcal{M}_{\lambda}\right), m \geq 1$, tell how 'lopsided' the moduli spaces are.

For the reduced moduli everything can be calculated explicitly. As shown in [5], the distortion $\Lambda: \partial \mathcal{K}_{0} \rightarrow \mathbf{R}$ is the maximal eigenvalue, and hence

$$
\frac{1}{\operatorname{dim} \mathcal{H}-1} \leq \Lambda \leq \operatorname{dim} \mathcal{H}-1
$$

It is easy to show that the reduced moduli $\mathcal{K}_{0}$ has a simplicial intersection in dimension $\operatorname{dim} \mathcal{H}-1$ corresponding to the diagonal endomorphisms with respect to an orthonormal basis in $\mathcal{H}$. Thus

$$
r\left(\mathcal{K}_{0}\right)=\operatorname{dim} \mathcal{H}-1 .
$$

Moreover, for $m \geq \operatorname{dim} \mathcal{H}$, we have

$$
\sigma_{m}\left(\mathcal{K}_{0}\right)=\frac{m+1}{\operatorname{dim} \mathcal{H}} .
$$


Clearly, for any linear subspace $\mathcal{E} \subset S_{0}^{2}(\mathcal{H})$, we also have

$$
r\left(\mathcal{K}_{0} \cap \mathcal{E}\right) \leq \operatorname{dim} \mathcal{H}-1 .
$$

Theorem C. If $\mathcal{L}=\mathcal{K}_{0} \cap \mathcal{E}$ for a linear subspace $\mathcal{E} \subset S_{0}^{2}(\mathcal{H})$, then the sequence $\left\{\sigma_{m}(\mathcal{L})\right\}_{m \geq r(\mathcal{L})}$ is strictly increasing. Moreover, we have

$$
r(\mathcal{L}) \leq \operatorname{dim} \mathcal{H}-n(\mathcal{L}),
$$

where

$$
n(\mathcal{L})=\min \{\operatorname{rank}(C+I) \mid C \in \partial \mathcal{L}\}(\geq 1) .
$$

If $r(\mathcal{L})=\operatorname{dim} \mathcal{H}-1$, then $\mathcal{E}$ contains all endomorphisms of $\mathcal{H}$ that are diagonal with respect to an orthonormal basis of $\mathcal{H}$, and the elements of the orthonormal basis form a minimal configuration for $\sigma_{\operatorname{dim} \mathcal{H}-1}(\mathcal{L})=1$.

Theorem $\mathrm{C}$ applies to the moduli $\mathcal{L}_{\lambda}$ and $\mathcal{M}_{\lambda}$ (both assumed to be nontrivial). Assuming that the first Betti number of $M$ is zero, we have $\operatorname{dim} V \geq 3$. (The topological condition guarantees that there is no nonconstant eigenmap of $M$ to the circle.) By the DoCarmo-Wallach parametrization, $\operatorname{rank}(C+I)$ corresponds to the range dimension of the eigenmap that $C$ parametrizes, and hence $n\left(\mathcal{L}_{\lambda}\right) \geq 3$. Theorem $\mathrm{C}$ now implies that

$$
r\left(\mathcal{L}_{\lambda}\right) \leq \operatorname{dim} \mathcal{H}_{\lambda}-3 .
$$

Note that it is a difficult problem to give better lower estimates of $n\left(\mathcal{L}_{\lambda}\right)$ in terms of $\lambda[6,7$.

For spherical minimal immersions $f: M \rightarrow S_{V}$ of a (positive) constant curvature domain $M$, a result of J.D. Moore [3] states that $\operatorname{dim} V \geq 2 \operatorname{dim} M$ (unless $f$ is totally geodesic, and therefore $\mathcal{M}_{\lambda}$ is trivial). Thus, Theorem $\mathrm{C}$ gives

$$
r\left(\mathcal{M}_{\lambda}\right) \leq \operatorname{dim} \mathcal{H}_{\lambda}-2 \operatorname{dim} M \text {. }
$$

We now return to the general setting. The distortion $\Lambda$ and (therefore) $\sigma_{m}(\mathcal{L})$ depend on the base point $\mathcal{O} \in \operatorname{int} \mathcal{L}$. Including this dependence, we obtain the distortion function $\Lambda: \partial \mathcal{L} \times \operatorname{int} \mathcal{L} \rightarrow \mathbf{R}$ and the invariant $\sigma_{m}(\mathcal{L},):$. int $\mathcal{L} \rightarrow \mathbf{R}$.

We have the following:

Theorem D. (a) $\Lambda: \partial \mathcal{L} \times \operatorname{int} \mathcal{L} \rightarrow \mathbf{R}$ is continuous; (b) $\sigma_{m}(\mathcal{L}$, .) is continuous on int $\mathcal{L}$ and extends continuously to $\partial \mathcal{L}$ by setting it as 1 on $\partial \mathcal{L}$.

Remark. If $\mathcal{L}$ is a square in a plane $\mathcal{E}$, then $\sigma_{m}(\mathcal{L},$.$) is not differentiable in int \mathcal{L}$.

By Theorems $\mathrm{A}$ and $\mathrm{D}, \sigma_{m}(\mathcal{L},$.$) attains its maximum at a point \mathcal{O}_{0}$ in the interior of $\mathcal{L}$. Although it may not be unique, it is natural to ask for a geometric characterization of $\mathcal{O}_{0}$ in terms of $\mathcal{L}$. (For example, if $\mathcal{L}$ is symmetric with respect to $\mathcal{O}_{0}$, then $\mathcal{O}_{0}$ is the centroid of $\mathcal{L}$, and $\sigma_{m}(\mathcal{L},$.$) attains its maximum value (m+1) / 2$ there.) In general, this question seems difficult. As a first step it is reasonable to expect that $\sigma_{m}(\mathcal{L},$.$) is a concave function on \mathcal{L}$. That this is true for $\operatorname{dim} \mathcal{L}=2$ is the content of the following:

Theorem E. Let $\operatorname{dim} \mathcal{L}=2$. Then $\sigma(\mathcal{L},$.$) is concave on \operatorname{int} \mathcal{L}$ and extends continuously to a concave function to $\mathcal{L}$.

In general, calculating $\sigma_{m}(\mathcal{L}), m \leq n$ (for any base point $\mathcal{O}$ ), is difficult even for plane polygons [4. The key question is whether one can restrict the infimum in (2) to configurations consisting of extremal points of $\mathcal{L}$. 
Recall that $C \in \partial \mathcal{L}$ is called extremal if $C$ is not contained in an open line segment that lies entirely in $\mathcal{L}$. We let $\mathcal{L}^{0}$ denote the set of extremal points of $\mathcal{L}$. We also let $\mathcal{C}_{m}^{0}(\mathcal{L})$ denote the set of all $m$-configurations of $\mathcal{L}$ whose points are all extremal in $\mathcal{L}$. By definition, $\left\{C_{0}, \ldots, C_{m}\right\} \in \mathcal{C}_{m}^{0}(\mathcal{L})$ if $\left\{C_{0}, \ldots, C_{m}\right\} \subset \mathcal{L}^{0}$ and $\mathcal{O} \in\left[C_{0}, \ldots, C_{m}\right]$. With this we define $\sigma_{m}^{0}(\mathcal{L})$ for $m \geq n$ by

$$
\sigma_{m}^{0}(\mathcal{L})=\inf _{\left\{C_{0}, \ldots, C_{m}\right\} \in \mathcal{C}_{m}^{0}(\mathcal{L})} \sum_{i=0}^{m} \frac{1}{1+\Lambda\left(C_{i}\right)} .
$$

As usual, we put $\sigma^{0}(\mathcal{L})=\sigma_{n}^{0}(\mathcal{L})$ and $\mathcal{C}^{0}(\mathcal{L})=\mathcal{C}_{n}^{0}(\mathcal{L})$, where $n=\operatorname{dim} \mathcal{L}$.

By the theorems of Carathéodory and Krein-Milman [1, $\mathcal{C}^{0}(\mathcal{L})$ and hence $\mathcal{C}_{m}^{0}(\mathcal{L})$, $m \geq n$, are nonempty. (Apply Carathéodory's theorem to $\mathcal{L}^{0}$ and use KreinMilman's theorem asserting that the convex hull of $\mathcal{L}^{0}$ is $\mathcal{L}$.) We see that $\sigma_{m}^{0}(\mathcal{L})$ exists for all $m \geq n$. For $m \leq n$ we define

$$
\sigma_{m}^{0}(\mathcal{L})=\inf _{\mathcal{O} \in \mathcal{F} \subset \mathcal{E}, \operatorname{dim} \mathcal{F}=m} \sigma^{0}(\mathcal{L} \cap \mathcal{F}),
$$

where the infimum is over affine subspaces $\mathcal{F} \subset \mathcal{E}$.

Note that a minimizing sequence for the infima in (10) and (11) may not subconverge, as $\mathcal{L}^{0}$ is not necessarily closed in $\mathcal{L}$.

Comparing (2)-(10) and (4)-(11), we obtain

$$
\sigma_{m}(\mathcal{L}) \leq \sigma_{m}^{0}(\mathcal{L})
$$

Being defined on a much more restricted class of configurations, $\sigma_{m}^{0}(\mathcal{L}), m \geq 1$, are easier to determine than $\sigma_{m}(\mathcal{L})$. For example, if $\mathcal{L}$ is a convex polytope, then $\mathcal{L}^{0}$ is the (finite) set of vertices, and $\sigma^{0}(\mathcal{L})$ can be determined by a finite enumeration.

It is easy to show that Theorem A holds when $\sigma_{m}(\mathcal{L})$ is replaced by $\sigma_{m}^{0}(\mathcal{L})$. Our next result states a connection between the two invariants as follows.

Theorem $\mathbf{F}$. Let $\mathcal{L}$ be a compact convex body in an $n$-dimensional Euclidean vector space $\mathcal{E}$ and $\mathcal{O}$ a base point in the interior of $\mathcal{L}$. Let $m \leq n$. Then either

$$
\sigma_{m}(\mathcal{L})=\sigma_{m}^{0}(\mathcal{L})
$$

or

$$
\sigma_{m}(\mathcal{L})=\sigma_{m-1}(\mathcal{L})+\frac{1}{1+\max _{\partial \mathcal{L}} \Lambda} .
$$

An immediate consequence of the theorem is to reduce the computation of $\sigma(\mathcal{L})$ for $\mathcal{L}$ a plane polygon, to a finite enumeration.

Corollary. Let $n=2$ and $\mathcal{L} \subset \mathcal{E}$ as in the theorem above. Then

$$
\sigma(\mathcal{L})=\min \left\{\sigma^{o}(\mathcal{L}), 1+\frac{1}{1+\max _{\partial \mathcal{L}} \Lambda}\right\} .
$$

Theorem $\mathrm{F}$ will be proved by an analysis of the local maxima of the distortion function $\Lambda: \partial \mathcal{L} \rightarrow \mathbf{R}$. As a byproduct, we will also obtain the following:

$$
\max _{\partial \mathcal{L}} \Lambda=\max _{\mathcal{L}^{0}} \Lambda .
$$




\section{Superadditivity of $\sigma_{m}(\mathcal{L})$}

In this section we prove Theorem B.

Consider a minimal $(m+k)$-configuration $\left\{C_{0}, \ldots, C_{m+k}\right\} \in \mathcal{C}_{m+k}(\mathcal{L})$. Since $\mathcal{O} \in\left[C_{0}, \ldots, C_{m+k}\right]$, we have

$$
\mathcal{O}=\sum_{i=0}^{m+k} \lambda_{i} C_{i}, \quad \sum_{i=0}^{m+k} \lambda_{i}=1,0 \leq \lambda_{i} \leq 1 .
$$

Consider the partial sum $\sum_{j=1}^{k} \lambda_{m+j} \geq 0$. If this is zero, then $\mathcal{O} \in\left[C_{0}, \ldots, C_{m}\right]$ and so $\left\{C_{0}, \ldots, C_{m}\right\} \in \mathcal{C}_{m}(\mathcal{L})$. By minimality, $\Lambda\left(C_{m+j}\right)=\max _{\partial \mathcal{L}} \Lambda, j=1, \ldots, k$. Using (2) and (3) repeatedly, we estimate

$$
\begin{aligned}
\sigma_{m+k}(\mathcal{L}) & =\sum_{i=0}^{m} \frac{1}{1+\Lambda\left(C_{i}\right)}+\frac{k}{1+\max _{\partial \mathcal{L}} \Lambda} \\
& =\sigma_{m}(\mathcal{L})+\frac{k}{1+\max _{\partial \mathcal{L}} \Lambda} \\
& \geq \sigma_{m+1}(\mathcal{L})+\frac{k-1}{1+\max _{\partial \mathcal{L}} \Lambda} \\
& \geq \sigma_{m+1}(\mathcal{L})+\sigma_{k}(\mathcal{L})-1,
\end{aligned}
$$

where in the last inequality we used $\sigma_{1}(\mathcal{L})=1$. This is $(8)$.

Thus, from now on we may assume that $\sum_{j=1}^{k} \lambda_{m+j}>0$. Let

$$
\mathcal{O}^{\prime}=\sum_{j=1}^{k} \frac{\lambda_{m+j}}{\lambda_{m+1}+\ldots+\lambda_{m+k}} C_{m+j} .
$$

We may also assume that $\mathcal{O}^{\prime} \neq \mathcal{O}$. Indeed, if $\mathcal{O}^{\prime}=\mathcal{O}$, then $\left\{C_{m+1}, \ldots, C_{m+k}\right\} \in$ $\mathcal{C}_{k-1}(\mathcal{L})$ and, by minimality, $\Lambda\left(C_{i}\right)=\max _{\partial \mathcal{L}} \Lambda, i=0, \ldots, m$. Using $(2)$ and $(3)$ again, we estimate

$$
\begin{aligned}
\sigma_{m+k}(\mathcal{L}) & =\frac{m+1}{1+\max _{\partial \mathcal{L}} \Lambda}+\sum_{j=1}^{k} \frac{1}{1+\Lambda\left(C_{m+j}\right)} \\
& =\frac{m+1}{1+\max _{\partial \mathcal{L}} \Lambda}+\sigma_{k-1}(\mathcal{L}) \\
& \geq \sigma_{m+1}(\mathcal{L})-1+\frac{1}{1+\max _{\partial \mathcal{L}} \Lambda}+\sigma_{k-1}(\mathcal{L}) \\
& \geq \sigma_{m+1}(\mathcal{L})+\sigma_{k}(\mathcal{L})-1
\end{aligned}
$$

where we used $\sigma_{1}(\mathcal{L})=1$. This is (8) again.

Finally, we assume that $\mathcal{O}^{\prime} \neq \mathcal{O}$. We let $C^{\prime} \in \partial \mathcal{L}$ be the intersection of the ray emanating from $\mathcal{O}$ and passing through $\mathcal{O}^{\prime}$ with $\partial \mathcal{L}$.

By (15), we have

$$
\mathcal{O}=\sum_{i=0}^{m} \lambda_{i} C_{i}+\left(\lambda_{m+1}+\ldots+\lambda_{m+k}\right) \mathcal{O}^{\prime} .
$$

Expressing $\mathcal{O}^{\prime}$ in terms of $C^{\prime}$, we obtain

$$
\left\{C_{0}, \ldots, C_{m}, C^{\prime}\right\} \in \mathcal{C}_{m+1}(\mathcal{L}) .
$$


On the other hand, since $\mathcal{O} \in\left[\mathcal{O}^{\prime}, C^{\prime o}\right]$ (and $\mathcal{O}^{\prime} \in\left[C_{m+1}, \ldots, C_{m+k}\right]$ ), we have

$$
\left\{C_{m+1}, \ldots, C_{m+k}, C^{o}\right\} \in \mathcal{C}_{k}(\mathcal{L}) .
$$

Using (16)-(17), we finally compute

$$
\begin{aligned}
\sigma_{m+k}(\mathcal{L}) & =\sum_{i=0}^{m} \frac{1}{1+\Lambda\left(C_{i}\right)}+\sum_{j=1}^{k} \frac{1}{1+\Lambda\left(C_{m+j}\right)} \\
& =\sum_{i=0}^{m} \frac{1}{1+\Lambda\left(C_{i}\right)}+\frac{1}{1+\Lambda\left(C^{\prime}\right)} \\
& +\sum_{j=1}^{k} \frac{1}{1+\Lambda\left(C_{m+j}\right)}+\frac{1}{1+\Lambda\left(C^{\prime o}\right)}-1 \\
& \geq \sigma_{m+1}(\mathcal{L})+\sigma_{k}(\mathcal{L})-1 .
\end{aligned}
$$

Theorem B follows.

\section{Moduli}

In this section we prove Theorem C. First, we make a few observations on the reduced moduli. For brevity, let $h=\operatorname{dim} \mathcal{H}$. For $v \in \mathcal{H},\|v\|=h$, we consider $C_{v}=v \odot v-I \in \partial \mathcal{K}_{0}$, where $\odot$ is the symmetric tensor product. The image of $C_{v}+I$ is $\mathbf{R} \cdot v \subset \mathcal{H}$. If $C \in \partial \mathcal{K}_{0}$ with $C+I$ having minimal image, then $C=C_{v}$ for some $v \in \mathcal{H},\|v\|=h$. It follows that the extremal points of $\mathcal{K}_{0}$ as a convex set are precisely the points $C_{v}$, for $v \in \mathcal{H},\|v\|=h$. (If $C$ is in the interior of a line segment $\left[C_{0}, C_{1}\right] \subset \partial \mathcal{K}_{0}$, then $\operatorname{im}(C+I)=\operatorname{im}\left(C_{0}+I\right)+\operatorname{im}\left(C_{1}+I\right)$.)

The distortion $\Lambda(C)$ of $C \in \partial \mathcal{K}_{0}$ is the largest eigenvalue of $C$ ]. For $C=C_{v}$ we have $\Lambda\left(C_{v}\right)=h-1$ with multiplicity 1 , and the other eigenvalue is -1 with multiplicity $h-1$. Hence maximal distortion of $\mathcal{K}_{0}$ is attained precisely at the points $C_{v}, v \in \mathcal{H},\|v\|=h$.

Let $\left\{e_{i}\right\}_{i=1}^{h} \subset \mathcal{H}$ be an orthonormal basis. By the above, a minimal $(h-1)$ configuration is $\left\{C_{\sqrt{h} e_{1}}, \ldots, C_{\sqrt{h} e_{h}}\right\} \subset \mathcal{C}_{h-1}\left(\mathcal{K}_{0}\right)$. We have

$$
\sigma_{h-1}\left(\mathcal{K}_{0}\right)=\sum_{i=1}^{h} \frac{1}{1+\Lambda\left(C_{\sqrt{h} e_{i}}\right)}=\sum_{i=1}^{h} \frac{1}{h}=1 .
$$

We obtain that $\mathcal{K}_{0}$ has a simplicial intersection in dimension $h-1$. Note that the simplicial intersection consists of the endomorphisms in $\mathcal{K}_{0} \subset S_{0}^{2}(\mathcal{H})$ that are diagonal with respect to the basis $\left\{e_{i}\right\}_{i=1}^{h}$.

For the beginning of the proof of the first statement of Theorem $\mathrm{C}$ on monotonicity, we keep $\mathcal{L}$ a general compact convex body $\mathcal{L} \subset \mathcal{E}$.

Assume that $\sigma_{k}(\mathcal{L})=\sigma_{k-1}(\mathcal{L})$ for some $k \geq 2$. Since $\sigma_{1}(\mathcal{L})=1$, we may assume that $k \geq 3$. Let $\left\{C_{0}, \ldots, C_{k}\right\} \in \mathcal{C}_{k}(\mathcal{L})$ be a minimizing $k$-configuration. We first show that $\left[C_{0}, \ldots, C_{k}\right]$ is a $k$-simplex and it is the intersection of $\mathcal{L}$ with the affine span of $\left\{C_{0}, \ldots, C_{k}\right\}$. Theorem A then gives $\sigma_{k}(\mathcal{L})=1$, that is, $k \leq r(\mathcal{L})$, and the first statement of the Corollary follows.

We first claim that $\left[C_{0}, \ldots, C_{k}\right]$ is a $k$-simplex with $\mathcal{O}$ in its relative interior.

If the dimension of the convex polytope $\left[C_{0}, \ldots, C_{k}\right]$ is less than $k$, or if $\left[C_{0}, \ldots\right.$, $\left.C_{k}\right]$ is a $k$-simplex but $\mathcal{O}$ is not in its relative interior, then $\mathcal{O}$ is in the convex hull 
of a proper subset of $\left\{C_{0}, \ldots, C_{k}\right\}$. We may assume that $\mathcal{O} \in\left[C_{0}, \ldots, C_{k_{0}}\right]$ for some $k_{0}<k$. By minimality, $\Lambda\left(C_{k_{0}+j}\right)=\max _{\partial \mathcal{L}} \Lambda$, for $j=1, \ldots, k-k_{0}$. We have

$$
\begin{aligned}
\sigma_{k}(\mathcal{L}) & =\sum_{j=0}^{k_{0}} \frac{1}{1+\Lambda\left(C_{j}\right)}+\frac{k-k_{0}}{1+\max _{\partial \mathcal{L}} \Lambda} \\
& =\sigma_{k-1}(\mathcal{L})+\frac{1}{1+\max _{\partial \mathcal{L}} \Lambda}>\sigma_{k-1}(\mathcal{L}) .
\end{aligned}
$$

This is a contradiction, and the claim follows.

Next, we claim that for $i, j=0, \ldots, k, i \neq j$, the affine plane $\mathcal{F}_{i j}$ spanned by $C_{i}, C_{j}$, and $\mathcal{O}$ intersects $\mathcal{L}$ in a triangle with vertices $C_{i}, C_{j}$ and another vertex. It is enough to show this for $i=0$ and $j=1$. We apply the construction in the proof of Theorem B (for $m=1$ and $k$ replaced by $k-1$ ). We arrive at the two configurations

$$
\left\{C_{0}, C_{1}, C^{\prime}\right\} \in \mathcal{C}_{2}(\mathcal{L})
$$

and

$$
\left\{C_{2}, \ldots, C_{k}, C^{\prime o}\right\} \in \mathcal{C}_{k-1}(\mathcal{L}),
$$

where $C^{\prime} \in \partial \mathcal{L}$ is on the ray emanating from $\mathcal{O}$ and passing through the point

$$
\mathcal{O}^{\prime}=\sum_{j=2}^{k} \frac{\lambda_{j}}{\lambda_{2}+\ldots+\lambda_{k}} C_{j} .
$$

We estimate

$$
\begin{aligned}
\sigma_{k}(\mathcal{L})= & \frac{1}{1+\Lambda\left(C_{0}\right)}+\frac{1}{1+\Lambda\left(C_{1}\right)}+\sum_{j=2}^{k} \frac{1}{1+\Lambda\left(C_{j}\right)} \\
= & \frac{1}{1+\Lambda\left(C_{0}\right)}+\frac{1}{1+\Lambda\left(C_{1}\right)}+\frac{1}{1+\Lambda\left(C^{\prime}\right)} \\
& +\sum_{j=2}^{k} \frac{1}{1+\Lambda\left(C_{j}\right)}+\frac{1}{1+\Lambda\left(C^{\prime o}\right)}-1 \\
\geq & \sigma_{2}(\mathcal{L})+\sigma_{k-1}(\mathcal{L})-1 .
\end{aligned}
$$

Since $\sigma_{k}(\mathcal{L})=\sigma_{k-1}(\mathcal{L})$ and $\sigma_{2}(\mathcal{L}) \geq 1$, equality holds. We obtain

$$
\sigma_{2}(\mathcal{L})=\frac{1}{1+\Lambda\left(C_{0}\right)}+\frac{1}{1+\Lambda\left(C_{1}\right)}+\frac{1}{1+\Lambda\left(C^{\prime}\right)}=1 .
$$

By Theorem A, $\mathcal{L} \cap \mathcal{F}_{01}=\left[C_{0}, C_{1}, C^{\prime}\right]$ is a triangle, and the claim follows.

The final step is to show that the affine span $\mathcal{F}$ of $\left\{C_{0}, \ldots, C_{k}\right\}$ intersects $\mathcal{L}$ in the $k$-simplex $\left[C_{0}, \ldots, C_{k}\right]$. Clearly, $\left[C_{0}, \ldots, C_{k}\right] \subset \mathcal{L} \cap \mathcal{F}$, and $\mathcal{L}$ can be replaced by $\mathcal{L} \cap \mathcal{F}$.

We now assume that $\mathcal{L}=\mathcal{K}_{0}(\mathcal{H}) \cap \mathcal{E}$, where $\mathcal{E} \subset S_{0}^{2}(\mathcal{H})$ is a linear subspace and $\mathcal{H}$ is a Euclidean vector space. We apply (ii) of Theorem $\mathrm{D}$ in 4 for any pair $C_{i}$ and $C_{j}$. Then the fact that $\mathcal{L} \cap \mathcal{F}_{i j}$ is a triangle is equivalent to the two conditions

$$
\operatorname{ker}\left(C_{i}+I\right) \cap \operatorname{ker}\left(C_{j}+I\right) \neq\{0\}
$$

and

$$
I-\frac{1}{1+\Lambda\left(C_{i}\right)}\left(C_{i}+I\right)-\frac{1}{1+\Lambda\left(C_{j}\right)}\left(C_{j}+I\right) \geq 0 .
$$


Let $v_{i}$ be an eigenvector corresponding to the maximal eigenvalue of $C_{i}$. Evaluating the last inequality on $v_{i}$, we obtain

$$
\left\langle\left(C_{j}+I\right) v_{i}, v_{i}\right\rangle \leq 0 .
$$

Since $C_{j}+I \geq 0, v_{i}$ is in the kernel of $C_{j}+I$. Since this is true for all $j \neq i$, we obtain that the intersection of the kernels of $C_{j}+I$ for all $j \neq i$ is nontrivial. This, however, means that $\left[C_{0}, \ldots, \widehat{C_{i}}, \ldots, C_{k}\right] \subset \partial \mathcal{L}$. Since this is true for all $i$, we have $\partial\left[C_{0}, \ldots, C_{k}\right] \subset \partial \mathcal{L}$, so that $\mathcal{L} \cap \mathcal{F}=\left[C_{0}, \ldots, C_{k}\right]$. The first statement of Theorem C follows.

Let $\mathcal{L}=\mathcal{K}_{0} \cap \mathcal{E}$ for some linear subspace $\mathcal{E} \subset S_{0}^{2}(\mathcal{H})$ and assume that $\sigma_{h-n}(\mathcal{L})=1$ for some $h>2$ and $1 \leq n<h-1$. Let $\left[C_{0}, \ldots, C_{h-n}\right]$ be a simplicial intersection of $\mathcal{K}_{0} \cap \mathcal{E}$, where $\left\{C_{0}, \ldots, C_{n-h}\right\} \in \mathcal{C}_{n-h}(\mathcal{L})$ is an $(h-n)$-minimal configuration. By (6)-(7), we have

$$
\sum_{i=0}^{n-h} \frac{1}{1+\Lambda\left(C_{i}\right)}\left(C_{i}+I\right)=I
$$

Let $V_{i} \subset \mathcal{H}$ denote the eigenspace corresponding to the (largest) eigenvalue $\Lambda\left(C_{i}\right)$ of $C_{i}$. By the equality (and the reasoning) above, $V_{i}$ is contained in the kernel of $C_{j}+I$ for any $j \neq i$. In particular, the eigenspaces $V_{i}$ are mutually orthogonal. We now estimate

$$
h-n \leq \sum_{i=0, i \neq j}^{h-n} \operatorname{dim} V_{i} \leq \operatorname{dim} \operatorname{ker}\left(C_{j}+I\right) \leq h-n(\mathcal{L}) .
$$

Thus $n \geq n(\mathcal{L})$, and the second statement of Theorem $\mathrm{C}$ follows.

For the proof of the last statement, assume that $n=1$. For reasons of dimension, we have $\sum_{i=0}^{h-1} V_{i}=\mathcal{H}, V_{i}$ is one dimensional, and $C_{i}$ has exactly two eigenvalues: $\Lambda\left(C_{i}\right)$ with multiplicity 1 and -1 with multiplicity $h-1$. Since trace $\left(C_{i}\right)=0$, we also have $\Lambda\left(C_{i}\right)=h-1$. Choosing a unit vector $e_{i} \in V_{i}$ for each $i=1, \ldots, h$, we get $C_{i}=C_{\sqrt{e}_{i}}=h e_{i} \odot e_{i}-I$. The last statement of Theorem $\mathrm{C}$ follows.

\section{Continuity of $\sigma_{m}(\mathcal{L},$.}

The following lemma asserts part (a) of Theorem D.

Lemma 1. (a) For fixed $\mathcal{O} \in \operatorname{int} \mathcal{L}$, the function $\Lambda=\Lambda(., \mathcal{O}): \partial \mathcal{L} \rightarrow \mathbf{R}$ is continuous; (b) The family of functions $\{\Lambda(C, .)\}_{C \in \partial \mathcal{L}}$ is equicontinuous; (c) $\Lambda$ : $\partial \mathcal{L} \times \operatorname{int} \mathcal{L} \rightarrow \mathbf{R}$ is continuous.

Proof. (a) This follows as in [1] but, for future purposes, we give here an independent proof. Let $C^{\prime} \rightarrow C$ in $\partial \mathcal{L}$. We need to show that $\Lambda\left(C^{\prime}, \mathcal{O}\right) \rightarrow \Lambda(C, \mathcal{O})$. Let $\alpha=\angle C O C^{\prime}$. The triangle $\triangle C \mathcal{O} C^{\prime}$ gives

$$
d\left(C^{\prime}, C\right)^{2}=\left(d\left(C^{\prime}, \mathcal{O}\right)-d(C, \mathcal{O})\right)^{2}+4 d\left(C^{\prime}, \mathcal{O}\right) d(C, \mathcal{O}) \sin ^{2}(\alpha / 2)
$$

Let $\delta_{\mathcal{O}}=\inf _{X \in \partial \mathcal{L}} d(X, \mathcal{O})$. Since $\mathcal{L}$ is compact, $\delta_{\mathcal{O}}>0$. By the equation above, we have $d\left(C^{\prime}, \mathcal{O}\right) \rightarrow d(C, \mathcal{O})$ and

$$
d\left(C^{\prime}, \mathcal{O}\right) d(C, \mathcal{O}) \sin ^{2}(\alpha / 2) \geq \delta_{\mathcal{O}}^{2} \sin ^{2}(\alpha / 2) \rightarrow 0 .
$$

We thus obtain $\alpha \rightarrow 0$. 
Let $C^{o}$ and $C^{\prime o}$ be the opposites of $C$ and $C^{\prime}$ (with respect to $\mathcal{O}$ ), respectively. We have $\alpha=\angle C^{o} \mathcal{O} C^{o}$. Let $\pi_{\mathcal{O}}: \partial \mathcal{L} \rightarrow S_{\mathcal{O}}$ be the radial projection of $\partial \mathcal{L}$ to the unit sphere $S_{\mathcal{O}}$ centered at $\mathcal{O}$. We have

$$
d\left(\pi_{\mathcal{O}}\left(C^{\prime o}\right), \pi_{\mathcal{O}}\left(C^{o}\right)\right)=2 \sin (\alpha / 2) \rightarrow 0 .
$$

$\pi_{\mathcal{O}}$ is a homeomorphism [1], so that $C^{\prime o} \rightarrow C^{o}$ follows.

Hence $d\left(C^{\prime o}, \mathcal{O}\right) \rightarrow d\left(C^{o}, \mathcal{O}\right)$ and we obtain

$$
\Lambda\left(C^{\prime}, \mathcal{O}\right)=\frac{d\left(C^{\prime}, \mathcal{O}\right)}{d\left(C^{\prime o}, \mathcal{O}\right)} \rightarrow \frac{d(C, \mathcal{O})}{d\left(C^{o}, \mathcal{O}\right)}=\Lambda(C, \mathcal{O})
$$

This is (a).

(b) Let $\mathcal{O}^{\prime} \rightarrow \mathcal{O}$ in int $\mathcal{L}, C$ a variable point on $\partial \mathcal{L}, C^{o}$ and $C^{\prime o}$ the opposites of $C$ with respect to $\mathcal{O}$ and $\mathcal{O}^{\prime}$, respectively. Let $\alpha=\angle \mathcal{O} C \mathcal{O}^{\prime}, \beta=\angle \mathcal{O} C^{\prime o} \mathcal{O}^{\prime}$ and $\gamma=\angle C^{\circ} \mathcal{O} C^{\prime o}$. All these angles depend on $C, \mathcal{O}$ and $\mathcal{O}^{\prime}$. Since $\gamma$ is an exterior angle to the triangle $\triangle \mathcal{O} C C^{\prime o}$, we have $\gamma=\alpha+\beta$. Let $\delta_{\mathcal{O}}=\inf _{X \in \partial \mathcal{L}} d(X, \mathcal{O})$ and $\Delta_{\mathcal{O}}=\sup _{X \in \partial \mathcal{L}} d(X, \mathcal{O})$. Then $\Delta_{\mathcal{O}} \geq \delta_{\mathcal{O}}>0$.

The triangle $\triangle \mathcal{O O}^{\prime} C$ gives

$$
d\left(\mathcal{O}^{\prime}, \mathcal{O}\right)^{2}=\left(d\left(C, \mathcal{O}^{\prime}\right)-d(C, \mathcal{O})\right)^{2}+4 d\left(C, \mathcal{O}^{\prime}\right) d(C, \mathcal{O}) \sin ^{2}(\alpha / 2),
$$

in particular,

$$
d\left(\mathcal{O}^{\prime}, \mathcal{O}^{\prime}\right)^{2} \geq 4 d\left(C, \mathcal{O}^{\prime}\right) d(C, \mathcal{O}) \sin ^{2}(\alpha / 2) .
$$

Let $0<\delta<\delta_{\mathcal{O}} / 2$ and assume that $d\left(\mathcal{O}^{\prime}, \mathcal{O}\right)<\delta$. Then $d\left(C, \mathcal{O}^{\prime}\right) \geq \delta_{\mathcal{O}}-\delta>\delta_{\mathcal{O}} / 2$ and the inequality above reduces to

$$
d\left(\mathcal{O}^{\prime}, \mathcal{O}\right) \geq \sqrt{2} \delta_{\mathcal{O}} \sin (\alpha / 2) .
$$

We obtain that $\alpha\left(C, \mathcal{O}, \mathcal{O}^{\prime}\right) \rightarrow 0$ as $\mathcal{O}^{\prime} \rightarrow \mathcal{O}$, uniformly in $C \in \partial \mathcal{L}$.

The triangle $\triangle C \mathcal{O} C^{\prime o}$ gives

$$
\sin \beta=\frac{d(C, \mathcal{O})}{d\left(C^{\prime o}, \mathcal{O}\right)} \sin \alpha \leq \frac{\Delta_{\mathcal{O}}}{\delta_{\mathcal{O}}} \sin \alpha .
$$

Hence $\beta\left(C, \mathcal{O}, \mathcal{O}^{\prime}\right) \rightarrow 0$ as $\mathcal{O}^{\prime} \rightarrow \mathcal{O}$, uniformly in $C \in \partial \mathcal{L}$.

Since $\gamma=\alpha+\beta, \gamma\left(C, \mathcal{O}, \mathcal{O}^{\prime}\right) \rightarrow 0$ as $\mathcal{O}^{\prime} \rightarrow \mathcal{O}$, uniformly in $C \in \partial \mathcal{L}$.

As in the first part of the proof

$$
d\left(\pi_{\mathcal{O}}\left(C^{\prime o}\right), \pi_{\mathcal{O}}\left(C^{o}\right)\right)=2 \sin (\gamma / 2)
$$

and we obtain that

$$
\pi_{\mathcal{O}}\left(C^{\prime o}\right) \rightarrow \pi_{\mathcal{O}}\left(C^{o}\right)
$$

as $\mathcal{O}^{\prime} \rightarrow \mathcal{O}$, uniformly in $C \in \partial \mathcal{L}$.

Once again, $\pi_{\mathcal{O}}$ is a homeomorphism (of compact sets), so that $C^{\prime o} \rightarrow C^{o}$ as $\mathcal{O}^{\prime} \rightarrow \mathcal{O}$ uniformly in $C \in \partial \mathcal{L}$.

Finally

and

$$
\left|d\left(C, \mathcal{O}^{\prime}\right)-d(C, \mathcal{O})\right| \leq d\left(\mathcal{O}^{\prime}, \mathcal{O}\right)
$$

$$
\begin{aligned}
\left|d\left(C^{\prime o}, \mathcal{O}^{\prime}\right)-d\left(C^{o}, \mathcal{O}\right)\right| & \leq\left|d\left(C^{\prime o}, \mathcal{O}^{\prime}\right)-d\left(C^{\prime o}, \mathcal{O}\right)\right|+\left|d\left(C^{\prime o}, \mathcal{O}\right)-d\left(C^{o}, \mathcal{O}\right)\right| \\
& \leq d\left(\mathcal{O}^{\prime}, \mathcal{O}\right)+d\left(C^{\prime o}, C^{o}\right),
\end{aligned}
$$

and both of these converge to zero uniformly in $C \in \partial \mathcal{L}$ as $\mathcal{O}^{\prime} \rightarrow \mathcal{O}$.

Combining these, we obtain that $\left|\Lambda(C, \mathcal{O})-\Lambda\left(C, \mathcal{O}^{\prime}\right)\right|$ converges to zero uniformly in $C \in \partial \mathcal{L}$ as $\mathcal{O}^{\prime} \rightarrow \mathcal{O}$. (b) follows.

Finally, (c) follows from (a) and (b). 
Let $\mathcal{O}, \mathcal{O}^{\prime} \in \operatorname{int} \mathcal{L}$ and $C \in \partial \mathcal{L}$. We define $\phi_{\mathcal{O}}\left(\mathcal{O}^{\prime}, C\right) \in \partial \mathcal{L}$ as the unique point such that $C-\mathcal{O}=\mu\left(\phi_{\mathcal{O}}\left(\mathcal{O}^{\prime}, C\right)-\mathcal{O}^{\prime}\right)$ for some (unique) $\mu>0$. We obtain the map $\phi_{\mathcal{O}}:$ int $\mathcal{L} \times \partial \mathcal{L} \rightarrow \partial \mathcal{L}$. Clearly, $\phi_{\mathcal{O}}(\mathcal{O}, C)=C$. In addition, for $\mathcal{O}, \mathcal{O}^{\prime}, \mathcal{O}^{\prime \prime} \in \operatorname{int} \mathcal{L}$, we have

$$
\phi_{\mathcal{O}^{\prime}}\left(\mathcal{O}^{\prime \prime}, \phi_{\mathcal{O}}\left(\mathcal{O}^{\prime}, C\right)\right)=\phi_{\mathcal{O}}\left(\mathcal{O}^{\prime \prime}, C\right)
$$

and consequently

$$
\phi_{\mathcal{O}^{\prime}}\left(\mathcal{O}, \phi_{\mathcal{O}}\left(\mathcal{O}^{\prime}, C\right)\right)=C
$$

Lemma 2. For fixed $C \in \partial \mathcal{L}, \phi_{\mathcal{O}}(., C): \operatorname{int} \mathcal{L} \rightarrow \partial \mathcal{L}$ is continuous.

Proof. By (18), it is enough to show continuity at $\mathcal{O}$. Let $\mathcal{O}^{\prime} \in \operatorname{int} \mathcal{L}$ and $\alpha=$ $\angle C \mathcal{O} \phi_{\mathcal{O}}\left(\mathcal{O}^{\prime}, C\right)$. Then, $\alpha=\angle \mathcal{O} \phi_{\mathcal{O}}\left(\mathcal{O}^{\prime}, C\right) \mathcal{O}^{\prime}$. Part (b) of the proof of Lemma 1 applied to the triangle $\triangle \mathcal{O O}^{\prime} \phi_{\mathcal{O}}\left(\mathcal{O}^{\prime}, C\right)$ gives

$$
d\left(\mathcal{O}, \mathcal{O}^{\prime}\right) \geq \sqrt{2} \delta_{\mathcal{O}} \sin (\alpha / 2), \quad \delta_{\mathcal{O}}=\inf _{X \in \partial \mathcal{L}} d(X, \mathcal{O})
$$

provided that $d\left(\mathcal{O}^{\prime}, \mathcal{O}\right)<\delta<\delta_{\mathcal{O}} / 2$. Thus, $\alpha \rightarrow 0$ as $\mathcal{O}^{\prime} \rightarrow \mathcal{O}$. On the other hand, part (a) of the proof of Lemma 1 applied to the triangle $\triangle C \mathcal{O} \phi_{\mathcal{O}}\left(\mathcal{O}^{\prime}, C\right)$ gives $\phi_{\mathcal{O}}\left(\mathcal{O}^{\prime}, C\right) \rightarrow C$. The lemma follows.

Lemma 3. Let $C_{0}, \ldots, C_{m} \in \partial \mathcal{L}$. Then

$$
\mathcal{O} \in\left[C_{0}, \ldots, C_{m}\right] \Longrightarrow \mathcal{O}^{\prime} \in\left[\phi_{\mathcal{O}}\left(\mathcal{O}^{\prime}, C_{0}\right), \ldots, \phi_{\mathcal{O}}\left(\mathcal{O}^{\prime}, C_{m}\right)\right]
$$

Proof. Let $\mathcal{O}=\sum_{i=0}^{m} \lambda_{i} C_{i}$, where $\sum_{i=0}^{m} \lambda_{i}=1,0 \leq \lambda_{i} \leq 1$. Let $\mu_{i}>0$ be such that $C_{i}-\mathcal{O}=\mu_{i}\left(\phi_{\mathcal{O}}\left(\mathcal{O}^{\prime}, C_{i}\right)-\mathcal{O}^{\prime}\right)$. Multiplying this by $\lambda_{i}$ and summing up with respect to $i$, we obtain

$$
\left(\sum_{i=0}^{m} \lambda_{i} \mu_{i}\right) \mathcal{O}^{\prime}=\sum_{i=0}^{m} \lambda_{i} \mu_{i} \phi_{\mathcal{O}}\left(\mathcal{O}^{\prime}, C_{i}\right) .
$$

The lemma follows.

Proof of Theorem D. We need to prove only part (b). Let $\mathcal{O} \in \operatorname{int} \mathcal{L}$ be fixed. We first prove continuity of $\sigma_{m}(\mathcal{L},$.$) at \mathcal{O}$. Let $\left\{C_{0}, \ldots, C_{m}\right\} \in \mathcal{C}_{m}(\mathcal{L}, \mathcal{O})$ be any $m$-configuration. By (c) of Lemma 1, the function

$$
\frac{1}{1+\Lambda}: \partial \mathcal{L} \times \operatorname{int} \mathcal{L} \rightarrow \mathbf{R}
$$

is continuous. Let $\epsilon>0$. Choose $\delta>0\left(\delta<\delta_{\mathcal{O}} / 2, \delta_{\mathcal{O}}=\inf _{X \in \partial \mathcal{L}} d(X, \mathcal{O})\right)$ such that $d\left(\mathcal{O}^{\prime}, \mathcal{O}\right)<\delta$ and $d\left(C^{\prime}, C\right)<\delta$ imply

$$
\left|\frac{1}{1+\Lambda\left(C^{\prime}, \mathcal{O}^{\prime}\right)}-\frac{1}{1+\Lambda(C, \mathcal{O})}\right|<\frac{\epsilon}{m+1} .
$$

Let $C_{i}^{\prime}=\phi_{\mathcal{O}}\left(\mathcal{O}^{\prime}, C_{i}\right)$. By Lemma 3 , we have $\left\{C_{0}^{\prime}, \ldots, C_{m}^{\prime}\right\} \in \mathcal{C}_{m}\left(\mathcal{L}, \mathcal{O}^{\prime}\right)$. Since $\phi_{\mathcal{O}}\left(., C_{i}\right)$ is continuous at $\mathcal{O}$ for every $i$, there exists $0<\delta^{\prime} \leq \delta$ such that $d\left(\mathcal{O}^{\prime}, \mathcal{O}\right)<$ $\delta^{\prime}$ implies $d\left(C_{i}^{\prime}, C_{i}\right)<\delta$ for all $i$. By $(19)$, for $d\left(\mathcal{O}^{\prime}, \mathcal{O}\right)<\delta^{\prime}$, we also have

$$
\left|\sum_{i=0}^{m} \frac{1}{1+\Lambda\left(C_{i}^{\prime}, \mathcal{O}^{\prime}\right)}-\sum_{i=0}^{m} \frac{1}{1+\Lambda\left(C_{i}, \mathcal{O}\right)}\right|<\epsilon .
$$

First, choose $\left\{C_{0}, \ldots, C_{m}\right\} \in \mathcal{C}_{m}(\mathcal{L}, \mathcal{O})$ to be $m$-minimal (with respect to $\mathcal{O}$ ):

$$
\sigma_{m}(\mathcal{L}, \mathcal{O})=\sum_{i=0}^{m} \frac{1}{1+\Lambda\left(C_{i}, \mathcal{O}\right)} .
$$


Substituting this into (20), we obtain

$$
\left|\sum_{i=0}^{m} \frac{1}{1+\Lambda\left(C_{i}^{\prime}, \mathcal{O}^{\prime}\right)}-\sigma_{m}(\mathcal{L}, \mathcal{O})\right|<\epsilon .
$$

Taking the infimum in the first sum over $\mathcal{C}_{m}\left(\mathcal{L}, \mathcal{O}^{\prime}\right)$, we obtain

$$
\sigma_{m}\left(\mathcal{L}, \mathcal{O}^{\prime}\right)-\sigma_{m}(\mathcal{L}, \mathcal{O})<\epsilon .
$$

Second, choose $\left\{C_{0}^{\prime}, \ldots, C_{m}^{\prime}\right\} \in \mathcal{C}_{m}\left(\mathcal{L}, \mathcal{O}^{\prime}\right)$ to be $m$-minimal (with respect to $\mathcal{O}^{\prime}$ ). This is possible by first choosing $C_{i}^{\prime}$ and then defining $C_{i}=\phi_{\mathcal{O}^{\prime}}\left(\mathcal{O}, C_{i}^{\prime}\right)$, since then $\phi_{\mathcal{O}}\left(\mathcal{O}^{\prime}, C_{i}\right)=C_{i}^{\prime}$. Again by $(20)$ we have

$$
\sum_{i=0}^{m} \frac{1}{1+\Lambda\left(C_{i}, \mathcal{O}\right)}-\sigma_{m}\left(\mathcal{L}, \mathcal{O}^{\prime}\right)<\epsilon .
$$

Taking the infimum in the first sum over $\mathcal{C}_{m}(\mathcal{L}, \mathcal{O})$, we obtain

$$
\sigma_{m}(\mathcal{L}, \mathcal{O})-\sigma_{m}\left(\mathcal{L}, \mathcal{O}^{\prime}\right)<\epsilon .
$$

Combining these, for $d\left(\mathcal{O}^{\prime}, \mathcal{O}\right)<\delta^{\prime}$ we have

$$
\left|\sigma_{m}\left(\mathcal{L}, \mathcal{O}^{\prime}\right)-\sigma_{m}(\mathcal{L}, \mathcal{O})\right|<\epsilon .
$$

Continuity of $\sigma_{m}(\mathcal{L},$.$) in int \mathcal{L}$ follows.

To finish the proof of the theorem we now derive the following relations:

$$
\lim _{d(\mathcal{O}, \partial \mathcal{L}) \rightarrow 0} \max _{X \in \partial \mathcal{L}} \Lambda(X, \mathcal{O})=\infty
$$

and

$$
\lim _{d(\mathcal{O}, \partial \mathcal{L}) \rightarrow 0} \sigma_{m}(\mathcal{L}, \mathcal{O})=1 .
$$

Changing the notation, we let $\mathcal{O} \in \operatorname{int} \mathcal{L}$ be fixed and $\epsilon>0$. To prove (21)-(22), we will show that, for any $\mathcal{O}^{\prime} \in \operatorname{int} \mathcal{L}, d\left(\mathcal{O}^{\prime}, \partial \mathcal{L}\right)<\epsilon$ (with $\epsilon$ small enough so that $\mathcal{O}^{\prime} \neq \mathcal{O}$ ) implies

$$
\frac{1}{\epsilon} \frac{\delta_{\mathcal{O}}^{2}}{\Delta_{\mathcal{O}}} \leq \max _{\partial \mathcal{L}} \Lambda\left(., \mathcal{O}^{\prime}\right)
$$

and

$$
(1 \leq) \sigma_{m}\left(\mathcal{L}, \mathcal{O}^{\prime}\right) \leq 1+\epsilon \frac{(m-1) \Delta_{\mathcal{O}}}{2 \delta_{\mathcal{O}}^{2}},
$$

where as usual $\delta_{\mathcal{O}}$ and $\Delta_{\mathcal{O}}$ are the minimum and maximum distances of a boundary point of $\mathcal{L}$ from $\mathcal{O}$.

Let $X \in \partial \mathcal{L}$ be the unique point on the extension of the half-line emanating from $\mathcal{O}$ and passing through $\mathcal{O}^{\prime}$. Let $C^{\prime} \in \partial \mathcal{L}$ be such that $d\left(C^{\prime}, \mathcal{O}^{\prime}\right)<\epsilon$ and let $C=$ $\phi_{\mathcal{O}^{\prime}}\left(\mathcal{O}, C^{\prime}\right)$. We may assume that $C^{\prime} \notin[X, \mathcal{O}]$. Finally, let $\mathcal{O}^{\prime \prime}=[X, \mathcal{O}] \cap\left[C^{\prime}, \mathcal{O}^{\prime}\right]$.

We have

$$
\frac{d\left(X, \mathcal{O}^{\prime}\right)}{d(X, \mathcal{O})}=\frac{d\left(\mathcal{O}^{\prime \prime}, \mathcal{O}^{\prime}\right)}{d(C, \mathcal{O})} \leq \frac{d\left(C^{\prime}, \mathcal{O}^{\prime}\right)}{d(C, \mathcal{O})}
$$

so that

$$
d\left(X, \mathcal{O}^{\prime}\right) \leq d\left(C^{\prime}, \mathcal{O}^{\prime}\right) \frac{d(X, \mathcal{O})}{d(C, \mathcal{O})} \leq d\left(C^{\prime}, \mathcal{O}^{\prime}\right) \frac{\Delta_{\mathcal{O}}}{\delta_{\mathcal{O}}} \leq \epsilon \frac{\Delta_{\mathcal{O}}}{\delta_{\mathcal{O}}}
$$


Hence

$$
\Lambda\left(X, \mathcal{O}^{\prime}\right)=\frac{d\left(X, \mathcal{O}^{\prime}\right)}{d\left(X^{o}, \mathcal{O}^{\prime}\right)} \leq \frac{d\left(X, \mathcal{O}^{\prime}\right)}{d\left(X^{o}, \mathcal{O}\right)} \leq \epsilon \frac{\Delta_{\mathcal{O}}}{\delta_{\mathcal{O}}^{2}},
$$

where the antipodal $X^{o}$ is with respect to $\mathcal{O}^{\prime}$. With this we have

$$
\Lambda\left(X^{o}, \mathcal{O}^{\prime}\right)=\frac{1}{\Lambda\left(X, \mathcal{O}^{\prime}\right)} \geq \frac{1}{\epsilon} \frac{\delta_{\mathcal{O}}^{2}}{\Delta_{\mathcal{O}}},
$$

and (23) follows.

To prove $(24)$, we first note that any antipodal pair of points on $\partial \mathcal{L}$ (with respect to $\left.\mathcal{O}^{\prime}\right)$ plus $X^{o}$ listed $(m-1)$-times constitutes an $m$-configuration for $\sigma\left(\mathcal{L}, \mathcal{O}^{\prime}\right)$. Since an antipodal pair of points contributes 1 to the sum in (2), we have

$$
\begin{aligned}
(1 \leq) \sigma_{m}\left(\mathcal{L}, \mathcal{O}^{\prime}\right) & \leq 1+\frac{m-1}{1+\Lambda\left(X^{o}, \mathcal{O}^{\prime}\right)} \\
& =1+(m-1) \frac{d\left(X, \mathcal{O}^{\prime}\right)}{d\left(X^{o}, X\right)} \\
& \leq 1+\epsilon \frac{(m-1) \Delta_{\mathcal{O}}}{2 \delta_{\mathcal{O}}},
\end{aligned}
$$

where we used (25) and the fact that $d\left(X^{o}, X\right) \geq 2 \delta_{\mathcal{O}}$ as $\mathcal{O} \in\left[X, X^{o}\right]$. (24) follows. The proof of Theorem D is complete.

\section{Concavity of $\sigma_{m}(\mathcal{L},$.}

We begin with the following:

Proposition 1. For fixed $C \in \partial \mathcal{L}$, the function $1 /(1+\Lambda(C,)$.$) is concave on int \mathcal{L}$.

Proof. Let $\mathcal{O}_{0}, \mathcal{O}_{1} \in \operatorname{int} \mathcal{L}$ and $0 \leq \lambda \leq 1$. Let $\mathcal{O}_{\lambda}=(1-\lambda) \mathcal{O}_{0}+\lambda \mathcal{O}_{1}$. The line passing through $C$ and $\mathcal{O}_{\lambda}$ intersects the segment $\left[C_{0}^{o}, C_{1}^{o}\right]$ at a point which we denote by $C_{\lambda}^{o}$. We then have

$$
C_{\lambda}^{o}=(1-\mu) C_{0}^{o}+\mu C_{1}^{o}
$$

for some $0 \leq \mu \leq 1$. Finally, we let

$$
\Lambda_{\lambda}=\frac{d\left(C, \mathcal{O}_{\lambda}\right)}{d\left(C_{\lambda}^{o}, \mathcal{O}_{\lambda}\right)},
$$

with $\Lambda_{0}$ and $\Lambda_{1}$ also defined when $\lambda=0$ and $\lambda=1$, respectively. For the distortions, we have

$$
\Lambda_{0}=\Lambda\left(C, \mathcal{O}_{0}\right) \text { and } \Lambda_{1}=\Lambda\left(C, \mathcal{O}_{1}\right)
$$

and

$$
\Lambda\left(C, \mathcal{O}_{\lambda}\right) \leq \Lambda_{\lambda}
$$

with equality iff $C_{\lambda}^{o}$ is a boundary point of $\mathcal{L}$.

To simplify the computations, it is convenient to introduce the notation

$$
N_{\lambda}=1+\frac{1}{\Lambda_{\lambda}} .
$$

With this, we have

$$
\begin{aligned}
N_{0}\left(\mathcal{O}_{0}-C\right) & =C_{0}^{o}-C \\
N_{1}\left(\mathcal{O}_{1}-C\right) & =C_{1}^{o}-C \\
N_{\lambda}\left(\mathcal{O}_{\lambda}-C\right) & =C_{\lambda}^{o}-C .
\end{aligned}
$$


In the third equation we use the expression of $C_{\lambda}^{o}$ in (26), split $C$ as $(1-\lambda) C+\lambda C$ and $(1-\mu) C+\mu C$, and use the first two equations to obtain

$$
N_{\lambda}\left((1-\lambda)\left(\mathcal{O}_{0}-C\right)+\lambda\left(\mathcal{O}_{1}-C\right)\right)=N_{0}(1-\mu)\left(\mathcal{O}_{0}-C\right)+N_{1} \mu\left(\mathcal{O}_{1}-C\right) .
$$

We may assume that $\mathcal{O}_{0}-C$ and $\mathcal{O}_{1}-C$ are linearly independent. We then obtain

$$
\begin{aligned}
N_{\lambda}(1-\lambda) & =N_{0}(1-\mu), \\
N_{\lambda} \lambda & =N_{1} \mu .
\end{aligned}
$$

Eliminating $\mu$ we get

$$
N_{\lambda}=\frac{N_{0} N_{1}}{N_{1}(1-\lambda)+N_{0} \lambda}
$$

Finally, expressing $N_{\lambda}$ in terms of $\Lambda_{\lambda}$, we have

$$
\frac{1}{1+\Lambda_{\lambda}}=\frac{1-\lambda}{1+\Lambda_{0}}+\frac{\lambda}{1+\Lambda_{1}} .
$$

Using (27)-(28) we finally arrive at

$$
\frac{1}{1+\Lambda\left(C, \mathcal{O}_{\lambda}\right)} \geq \frac{1-\lambda}{1+\Lambda\left(C, \mathcal{O}_{0}\right)}+\frac{\lambda}{1+\Lambda\left(C, \mathcal{O}_{1}\right)}
$$

The proposition follows.

Corollary. The function

$$
\frac{1}{1+\max _{C \in \partial \mathcal{L}} \Lambda(C, .)}
$$

is continuous and concave in int $\mathcal{L}$, and it extends to $\mathcal{L}$ as a continuous concave function by setting it as zero on $\partial \mathcal{L}$.

Proof. Consider the family of functions

$$
\left\{\frac{1}{1+\Lambda(C, .)}\right\}_{C \in \partial \mathcal{L}}
$$

The (pointwise) minimum of this family is (29). By part (b) of Lemma 1 in Section 2 , this family is equicontinuous. Continuity of the minimum follows easily. Since the pointwise minimum of concave functions is concave, concavity of (29) also follows. Finally the second statement follows from (21).

We now prove a partial concavity of $\sigma_{m}(\mathcal{L},$.$) restricted to m$-minimizing configurations.

Lemma 1. Let $\left\{C_{0}, \ldots, C_{m}\right\} \in \mathcal{C}_{m}(\mathcal{L}, \mathcal{O})$ be an m-minimal configuration for $\sigma_{m}(\mathcal{L}, \mathcal{O})$. Then, for any $\mathcal{O}_{0}, \mathcal{O}_{1} \in\left[C_{0}, \ldots, C_{m}\right]$ and $0 \leq \lambda \leq 1$ such that $\mathcal{O}=$ $(1-\lambda) \mathcal{O}_{0}+\lambda \mathcal{O}_{1}$, we have

$$
\sigma_{m}(\mathcal{L}, \mathcal{O}) \geq(1-\lambda) \sigma_{m}\left(\mathcal{L}, \mathcal{O}_{0}\right)+\lambda \sigma_{m}\left(\mathcal{L}, \mathcal{O}_{1}\right)
$$

Proof. This is a consequence of Proposition 1. In fact, we have

$$
\sigma_{m}(\mathcal{L}, \mathcal{O})=\sum_{i=0}^{m} \frac{1}{1+\Lambda\left(C_{i}, \mathcal{O}\right)} \geq \sum_{i=0}^{m} \frac{(1-\lambda)}{1+\Lambda\left(C_{i}, \mathcal{O}_{0}\right)}+\sum_{i=0}^{m} \frac{\lambda}{1+\Lambda\left(C_{i}, \mathcal{O}_{1}\right)},
$$

since each term in the sum of the left-hand side is concave. Now, by assumption, $\left\{C_{0}, \ldots, C_{m}\right\} \in \mathcal{C}_{m}\left(\mathcal{L}, \mathcal{O}_{0}\right)$ and $\left\{C_{0}, \ldots, C_{m}\right\} \in \mathcal{C}_{m}\left(\mathcal{L}, \mathcal{O}_{0}\right)$ so that the infimums on the right-hand side sums can be taken over $\mathcal{C}_{m}\left(\mathcal{L}, \mathcal{O}_{0}\right)$ and $\mathcal{C}_{m}\left(\mathcal{L}, \mathcal{O}_{1}\right)$, respectively. The lemma follows. 
The principal difficulty in proving concavity of $\sigma_{m}(\mathcal{L},$.$) lies in the fact that$ $\left[\mathcal{O}_{0}, \mathcal{O}_{1}\right]$ may not be contained in any $m$-minimizing configuration. To circumvent this difficulty one is tempted to consider the recursive inequality

$$
\sigma_{m}(\mathcal{L}, \mathcal{O}) \leq \sigma_{m-1}(\mathcal{L}, \mathcal{O})+\frac{1}{1+\max _{C \in \partial \mathcal{L}} \Lambda(C, \mathcal{O})}
$$

and split the interior of $\mathcal{L}$ into two subsets according to whether equality or inequality holds. This approach works for $\operatorname{dim} \mathcal{L}=m=2$, and we assume this for the rest of this section (and suppress $m$ ).

We define two sets

$$
\mathcal{R}=\left\{\mathcal{O} \in \operatorname{int} \mathcal{L} \mid \sigma(\mathcal{L}, \mathcal{O})<1+\frac{1}{1+\max _{C \in \partial \mathcal{L}} \Lambda(C, \mathcal{O})}\right\}
$$

and

$$
\mathcal{S}=\left\{\mathcal{O} \in \operatorname{int} \mathcal{L} \mid \sigma(\mathcal{L}, \mathcal{O})=1+\frac{1}{1+\max _{C \in \partial \mathcal{L}} \Lambda(C, \mathcal{O})}\right\}
$$

By continuity of the functions involved, $\mathcal{R}$ is open and $\mathcal{S}$ is relatively closed in int $\mathcal{L}$.

Lemma 2. Let $\mathcal{O} \in \mathcal{S}$. Then the sets

$$
\mathcal{M}_{\mathcal{O}}=\left\{C \in \partial \mathcal{L} \mid \Lambda(C, \mathcal{O})=\max _{X \in \partial \mathcal{L}} \Lambda(X, \mathcal{O})\right\}
$$

and

$$
\mathcal{N}_{\mathcal{O}}=\left\{C \in \partial \mathcal{L} \mid \Lambda(C, \mathcal{O})=\min _{X \in \partial \mathcal{L}} \Lambda(X, \mathcal{O})\right\}
$$

are connected (and closed) and centrally symmetric with respect to $\mathcal{O}$. If $\Lambda$ is nonconstant, then $\Lambda$ decreases from $\mathcal{M}_{\mathcal{O}}$ to $\mathcal{N}_{\mathcal{O}} .\left\{C_{0}, C_{1}, C_{2}\right\} \in \mathcal{C}(\mathcal{L}, \mathcal{O})$ is minimal iff either one element is in $\mathcal{M}_{\mathcal{O}}$ and the other two are antipodal, or two elements are in $\mathcal{M}_{\mathcal{O}}$ and one is in $\mathcal{N}_{\mathcal{O}}$.

Proof. Since $\mathcal{O} \in \mathcal{S}$, for any configuration $\left\{C_{0}, C_{1}, C_{2}\right\} \in \mathcal{C}(\mathcal{L}, \mathcal{O})$, we have

$$
1+\frac{1}{1+\max _{C \in \partial \mathcal{L}} \Lambda(C, \mathcal{O})} \leq \frac{1}{1+\Lambda\left(C_{0}, \mathcal{O}\right)}+\frac{1}{1+\Lambda\left(C_{1}, \mathcal{O}\right)}+\frac{1}{1+\Lambda\left(C_{2}, \mathcal{O}\right)}
$$

Let $C_{0} \in \mathcal{M}_{\mathcal{O}}$ and $C_{2}=C_{1}^{o}$ and move $C_{1}$ to $C_{1}^{\prime}$ along $\partial \mathcal{L}$ keeping $\mathcal{O} \in\left[C_{0}, C_{1}, C_{2}\right]$. Then (30) implies $\Lambda\left(C_{1}^{\prime}\right) \leq \Lambda\left(C_{1}\right)$. Since this is true for any $C_{1}$ (between $C_{0}$ and $C_{0}^{o}$ ), the description of $\Lambda$ applies. The rest is clear.

Corollary. If $\mathcal{O} \in \mathcal{R}$ and $\left\{C_{0}, C_{1}, C_{2}\right\} \in \mathcal{C}(\mathcal{L}, \mathcal{O})$ is minimal, then $\left[C_{0}, C_{1}, C_{2}\right]$ is a (nonsingular) triangle with $\mathcal{O}$ in its interior.

For each $\mathcal{O} \in \mathcal{R}$, choose a minimal triangle $\left\{C_{0}, C_{1}, C_{2}\right\} \in \mathcal{C}(\mathcal{L}, \mathcal{O})$, and let

$$
\rho_{\mathcal{O}}=\inf _{X \in \partial\left[C_{0}, C_{1}, C_{2}\right]} d(X, \mathcal{O})>0 .
$$

Lemma 3. Let $K \subset \mathcal{R}$ be compact. Then $\inf _{\mathcal{O} \in K} \rho_{\mathcal{O}}>0$.

Proof. Assume, on the contrary, that there exist $\mathcal{O}_{n} \in K$ such that $\rho_{\mathcal{O}_{n}} \rightarrow 0$ as $n \rightarrow \infty$. Passing to a subsequence, we may assume that $\mathcal{O}_{n} \rightarrow \mathcal{O} \in K$. Since $\partial \mathcal{L}$ is also compact, we may also assume that each vertex of the minimizing triangle associated to $\mathcal{O}_{n}$ converges as $n \rightarrow \infty$. This way, we obtain a limiting configuration $\left\{C_{0}, C_{1}, C_{2}\right\} \in \mathcal{C}(\mathcal{L}, \mathcal{O})$. Since $\sigma(\mathcal{L},$.$) is continuous (part (b) of Theorem D),$ $\sigma\left(\mathcal{L}, \mathcal{O}_{n}\right) \rightarrow \sigma(\mathcal{L}, \mathcal{O})$ as $\mathcal{O}_{n} \rightarrow \mathcal{O}$. Since $\Lambda$ is continuous (part (a) of Theorem D), 
$\left\{C_{0}, C_{1}, C_{2}\right\}$ is minimal with respect to $\mathcal{O}$. On the other hand, $\rho_{\mathcal{O}}=0$. This is a contradiction since $\mathcal{O} \in \mathcal{R}$.

Proposition 2. $\sigma(\mathcal{L},$.$) is concave on \mathcal{R}$, that is, for $\left[\mathcal{O}_{0}, \mathcal{O}_{1}\right] \subset \mathcal{R}$ and $0 \leq \lambda \leq 1$, we have

$$
\sigma\left(\mathcal{L},(1-\lambda) \mathcal{O}_{0}+\lambda \mathcal{O}_{1}\right) \geq(1-\lambda) \sigma\left(\mathcal{L}, \mathcal{O}_{0}\right)+\lambda \sigma\left(\mathcal{L}, \mathcal{O}_{1}\right) .
$$

Proof. Let $\ell$ be the line passing through $\mathcal{O}_{0}$ and $\mathcal{O}_{1}$. Restricting $\sigma(\mathcal{L},$.$) to the$ connected component of $\mathcal{R} \cap \ell$ that contains $\left[\mathcal{O}_{o}, \mathcal{O}_{1}\right]$ and using Lemma 1 and Lemma 3 , the proposition is the consequence of the following.

Lemma 4. Let $f:\left(a_{0}, b_{0}\right) \rightarrow \mathbf{R}$ be a continuous function that satisfies the following property:

For each $c \in\left(a_{0}, b_{0}\right)$ there exists $\epsilon_{c}>0$ such that $\left(c-\epsilon_{c}, c+\epsilon_{c}\right) \subset\left(a_{0}, b_{0}\right)$ and for any $\left[c_{0}, c_{1}\right] \subset\left(c-\epsilon_{c}, c+\epsilon_{c}\right)$ with $c \in\left[c_{0}, c_{1}\right]$, we have

$$
f(c) \geq(1-\lambda) f\left(c_{0}\right)+\lambda f\left(c_{1}\right),
$$

where $c=(1-\lambda) c_{0}+\lambda c_{1}$. Moreover, given $[a, b] \subset\left(a_{0}, b_{0}\right)$, there is a universal choice of $\epsilon$ for all $c \in[a, b]$.

Then $f$ is concave on $\left(a_{0}, b_{0}\right)$.

Proof. Let $[a, b] \subset\left(a_{0}, b_{0}\right)$ and $\epsilon$ the universal choice for $[a, b]$. Let $0<\lambda<1$. We need to show that

$$
f((1-\lambda) a+\lambda b) \geq(1-\lambda) f(a)+\lambda f(b) .
$$

Let $c=(1-\lambda) a+\lambda b$. Let $N$ be a natural number and subdivide $[a, c]$ and $[c, b]$ into $N+1$ equal parts. Let the subdivision points be

$$
a=a_{0}<a_{1}<\ldots<a_{N}<a_{N+1}=c=b_{N+1}<b_{N}<\ldots<b_{1}<b_{0}=b .
$$

Note that $c=(1-\lambda) a_{i}+\lambda b_{i}, i=0, \ldots, N$. Choose $N$ large enough so that the $\epsilon$-neighborhood of any subdivision point inside $(a, b)$ contains at least three subdivision points. We now write down (31) for each of the three subdivision points:

$$
\begin{aligned}
f\left(a_{i}\right) & \geq \frac{1}{2} f\left(a_{i-1}\right)+\frac{1}{2} f\left(a_{i+1}\right), i=1, \ldots, N \\
f(c) & \geq(1-\lambda) f\left(a_{N}\right)+\lambda f\left(b_{N}\right), \\
f\left(b_{i}\right) & \geq \frac{1}{2} f\left(b_{i+1}\right)+\frac{1}{2} f\left(b_{i-1}\right), i=1, \ldots, N .
\end{aligned}
$$

We refer to the $i$-th inequality in the first set of $N$ inequalities as $(A)_{i}$, and the $i$-th inequality in the last set of $N$ inequalities as $(B)_{i}$, and the inequality for $f(c)$ as the middle inequality. (Note the apparent symmetry between $(A)_{i}$ and $(B)_{i}$.) We now claim that

$$
f(c) \geq(1-\lambda) f\left(a_{N-i}\right)+\lambda f\left(b_{N-i}\right), \quad i=0, \ldots, N .
$$

For $i=N$, this is (32). To prove (33) inductively, we first note that, for $i=0$, this is the middle equation. For the general induction step $i \Rightarrow i+1$, we consider (with obvious notations) the inequality

$$
(1-\lambda)\left((A)_{N-i}+\ldots+(A)_{N}\right)+\lambda\left((B)_{N-i}+\ldots+(B)_{N}\right)
$$


which, after cancellations, becomes

$$
\begin{aligned}
(1-\lambda)\left(f\left(a_{N-i}\right)+f\left(a_{N}\right)\right)+\lambda\left(f\left(b_{N-i}\right)+f\left(b_{N}\right)\right) & \\
& \geq(1-\lambda) f\left(a_{N-i-1}\right)+\lambda f\left(b_{N-i-1}\right)+f(c) .
\end{aligned}
$$

The left-hand side can be estimated from above by $2 f(c)$ by (33) and the middle inequality. (33) for $i+1$ follows, and the induction is complete.

Summarizing, we arrive at the following scenario:

On the open set $\mathcal{R}, \sigma(\mathcal{L},$.$) is concave and less than 1+1 /\left(1+\max _{\partial \mathcal{L}} \Lambda\right)$, and on $\mathcal{S}, \sigma(\mathcal{L},$.$) is equal to 1+1 /\left(1+\max _{\partial \mathcal{L}} \Lambda\right)$, and this latter function is concave on the entire $\mathcal{L}$.

To show that $\sigma(\mathcal{L},$.$) is concave, we consider \left[\mathcal{O}_{0}, \mathcal{O}_{1}\right] \subset \operatorname{int} \mathcal{L}$ and its extension to the line $\ell$. We let $f$ and $g$ denote the restrictions of $1+1 /\left(1+\max _{\partial \mathcal{L}} \Lambda\right)$ and $\sigma(\mathcal{L},$.$) to \ell \cap \mathcal{L} . g$ differs from $f$ on $U=\mathcal{R} \cap \ell$ which is a countable union of disjoint open intervals. Concavity of $\sigma(\mathcal{L},$.$) is now implied by the following.$

Lemma 5. Let $f:[a, b] \rightarrow \mathbf{R}$ be a continuous and concave function with $f(a)=$ $f(b)=1$. Let $U \subset(a, b)$ be an open set. Write $U$ as a countable union of disjoint open intervals $\left(a_{i}, b_{i}\right), i=1,2, \ldots$ For each $i$, let $g_{i}:\left[a_{i}, b_{i}\right] \rightarrow \mathbf{R}$ be concave, $g_{i}<f$ on $\left(a_{i}, b_{i}\right), g_{i}\left(a_{i}\right)=f\left(a_{i}\right)$ and $g_{i}\left(b_{i}\right)=f\left(b_{i}\right)$. Define $g:[a, b] \rightarrow \mathbf{R}$ as $g_{i}$ on $\left[a_{i}, b_{i}\right]$ for all $i$ and $g=f$ otherwise. Then $g$ is concave.

Proof. We let $f_{0}=f$. We define $f_{1}$ as $f_{0}$ replaced by $g_{1}$ on $\left[a_{1}, b_{1}\right]$. Proceeding inductively, we define $f_{i}$ as $f_{i-1}$ replaced by $g_{i}$ on $\left[a_{i}, b_{i}\right]$. Since the subintervals in question are disjoint, the (decreasing) sequence $f_{i}, i=1,2, \ldots$, is pointwise convergent and converges to $g$. It remains to show that $f_{i}$ is concave for each $i$. Proceeding inductively again, we need to show that concavity of $f_{i-1}$ implies concavity of $f_{i}$. Let $\left[c_{0}, c_{1}\right] \subset[a, b]$. We need to show that

$$
f_{i}\left((1-\lambda) c_{0}+\lambda c_{1}\right) \geq(1-\lambda) f\left(c_{0}\right)+\lambda f_{i}\left(c_{1}\right), 0 \leq \lambda \leq 1 .
$$

This is an easy case-by-case verification depending on the mutual position of $\left[c_{0}, c_{1}\right]$ and the interval $\left[a_{i}, b_{i}\right]$ on which $f_{i-1}$ is modified to $f_{i}$. The lemma follows.

\section{The invariants $\sigma_{m}^{0}(\mathcal{L})$}

In this section we prove an analogue of the theorem on $\sigma_{m}(\mathcal{L})$ for the invariants $\sigma_{m}^{0}(\mathcal{L})$ as follows.

Proposition. Let $\mathcal{L}$ be a compact convex body in an $n$-dimensional Euclidean vector space $\mathcal{E}$ and $\mathcal{O}$ a base point in the interior of $\mathcal{L}$. Let $m \geq 1$. Then

$$
1 \leq \sigma_{m}^{0}(\mathcal{L}) \leq \frac{m+1}{2} .
$$

If $\sigma_{m}^{0}(\mathcal{L})=1$, then $m \leq n$, and there exists an affine subspace $\mathcal{F} \subset \mathcal{E}, \mathcal{O} \in \mathcal{F}$, of dimension $m$ such that $\mathcal{L} \cap \mathcal{F}$ is an m-simplex. In this case a minimal configuration $\left\{C_{0}, \ldots, C_{m}\right\} \in \mathcal{C}^{0}(\mathcal{L} \cap \mathcal{F})$ is unique and is given by the set of vertices of $\mathcal{L} \cap \mathcal{F}$. Moreover, minimality

$$
\sum_{i=0}^{m} \frac{1}{1+\Lambda\left(C_{i}\right)}=1
$$

implies

$$
\sum_{i=0}^{m} \frac{1}{1+\Lambda\left(C_{i}\right)} C_{i}=0
$$


Conversely, if $\mathcal{L}$ has a simplicial intersection with an $m$-dimensional affine subspace $\mathcal{F} \ni \mathcal{O}$, then $\sigma_{m}^{0}(\mathcal{L})=1$.

For $m \geq 2, \sigma_{m}^{0}(\mathcal{L})=(m+1) / 2$ iff $\Lambda=1$ on $\partial \mathcal{L}$, that is, iff $\mathcal{L}$ is symmetric.

Proof. Combining (5) and $(12)$ we have $1 \leq \sigma_{m}(\mathcal{L}) \leq \sigma_{m}^{0}(\mathcal{L})$. If $\sigma_{m}^{0}(\mathcal{L})=1$, then $\sigma_{m}(\mathcal{L})=1$, and Theorem A implies that $\mathcal{L}$ has a simplicial intersection with an $m$-dimensional affine subspace $\mathcal{F} \ni \mathcal{O}$. In addition, minimal configuration exists and is unique and satisfies (6)-(7).

Conversely, if $\mathcal{L}$ has a simplicial intersection with an $m$-dimensional affine subspace $\mathcal{F} \ni \mathcal{O}$, then the vertices of the $m$-simplex $\mathcal{L} \cap \mathcal{F}$ are extremal points. Thus, $\sigma^{0}(\mathcal{L} \cap \mathcal{F})=1$ and so $\sigma_{m}^{0}(\mathcal{L})=1$.

It remains to prove the statements on the upper bound for $\sigma^{0}(\mathcal{L})$.

We first show the following:

$$
\sigma_{m+k}^{0}(\mathcal{L}) \leq \sigma_{m}^{0}(\mathcal{L})+\frac{k}{1+\max _{\mathcal{L}^{0} \Lambda}}, \quad k \geq 0 .
$$

We may assume that $k>1$. By (14) we can choose $C \in \mathcal{L}^{0}$ such that

$$
\Lambda(C)=\max _{\partial \mathcal{L}} \Lambda=\max _{\mathcal{L}^{0}} \Lambda \text {. }
$$

First let $m \geq n$. Let $\epsilon>0$ and choose $\left\{C_{0}, \ldots, C_{m}\right\} \in \mathcal{C}_{m}^{0}(\mathcal{L})$ such that

$$
\sum_{i=0}^{m} \frac{1}{1+\Lambda\left(C_{i}\right)}<\sigma_{m}^{0}(\mathcal{L})+\epsilon
$$

Adding $k$ copies of $C$ to the original configuration we obtain the $(m+k)$-configuration $\left\{C_{0}, \ldots, C_{m}, C, \ldots, C\right\} \in \mathcal{C}_{m+k}^{0}(\mathcal{L})$. By definition, we have

$$
\sigma_{m+k}^{0}(\mathcal{L}) \leq \sum_{i=0}^{m} \frac{1}{1+\Lambda\left(C_{i}\right)}+\frac{k}{1+\Lambda(C)}<\sigma_{m}^{0}(\mathcal{L})+\epsilon+\frac{k}{1+\Lambda(C)}
$$

Letting $\epsilon \rightarrow 0$, (34) follows.

Now let $m \leq n$. Let $\mathcal{F} \subset \mathcal{E}$ be an $m$-dimensional affine subspace with $\mathcal{O} \in \mathcal{F}$ such that

$$
\sigma^{0}(\mathcal{L} \cap \mathcal{F})<\sigma_{m}^{0}(\mathcal{L})+\epsilon
$$

Let $\left\{C_{0}, \ldots, C_{m}\right\} \in \mathcal{C}^{0}(\mathcal{L} \cap \mathcal{F})$ be such that

$$
\sum_{i=0}^{m} \frac{1}{1+\Lambda\left(C_{i}\right)}<\sigma^{0}(\mathcal{L} \cap \mathcal{F})+\epsilon
$$

Combining these, we obtain

$$
\sum_{i=0}^{m} \frac{1}{1+\Lambda\left(C_{i}\right)}<\sigma_{m}^{0}(\mathcal{L})+2 \epsilon
$$

If $k \geq n-m$, then, adding $k$ copies of $C$ again, we obtain $\left\{C_{0}, \ldots, C_{m}, C, \ldots, C\right\} \in$ $\mathcal{C}_{m+k}^{0}(\mathcal{L})$, and we have

$$
\sigma_{m+k}^{0}(\mathcal{L}) \leq \sum_{i=0}^{m} \frac{1}{1+\Lambda\left(C_{i}\right)}+\frac{k}{1+\Lambda(C)}<\sigma_{m}^{0}(\mathcal{L})+2 \epsilon+\frac{k}{1+\Lambda(C)} .
$$

Letting $\epsilon \rightarrow 0$, (34) follows. 
Finally, if $k<n-m$, then let $\mathcal{F}^{\prime} \supset \mathcal{F}$ be an $(m+k)$-dimensional affine subspace such that $C \in \mathcal{F}^{\prime}$. Then adding $k$ copies of $C$ again, we obtain

$$
\left\{C_{0}, \ldots, C_{m}, C, \ldots, C\right\} \in \mathcal{C}_{m+k}^{0}\left(\mathcal{L} \cap \mathcal{F}^{\prime}\right) .
$$

By definition, we have

$$
\begin{aligned}
\sigma_{m+k}^{0}(\mathcal{L}) & \leq \sigma^{0}\left(\mathcal{L} \cap \mathcal{F}^{\prime}\right) \\
& \leq \sum_{i=0}^{m} \frac{1}{1+\Lambda\left(C_{i}\right)}+\frac{k}{1+\Lambda(C)} \\
& <\sigma_{m}^{0}(\mathcal{L})+2 \epsilon+\frac{k}{1+\Lambda(C)}
\end{aligned}
$$

Letting $\epsilon \rightarrow 0$, (34) follows.

For $m=n=\operatorname{dim} \mathcal{L}$, we claim that equality holds in (34), so that the sequence $\left\{\sigma_{m}^{0}(\mathcal{L})\right\}_{m \geq n}$ is arithmetic with difference $1 /\left(1+\max _{\mathcal{L}^{0}} \Lambda\right)$. Indeed, let $\left\{C_{0}, \ldots, C_{n+k}\right\} \in \mathcal{C}_{n+k}^{0}(\mathcal{L})$ be such that

$$
\sum_{i=0}^{n+k} \frac{1}{1+\Lambda\left(C_{i}\right)}<\sigma_{n+k}^{0}(\mathcal{L})+\epsilon
$$

The convex hull $\left[C_{0}, \ldots, C_{n+k}\right] \ni \mathcal{O}$ is a convex polytope of dimension $\leq n$ since it is contained in $\mathcal{E}$. We can select $n+1$ points from $\left\{C_{0}, \ldots, C_{n+k}\right\}$ whose convex hull still contains $\mathcal{O}$. Renumbering if necessary, we may assume that these are $C_{0}, \ldots, C_{n}$ so that $\mathcal{O} \in\left[C_{0}, \ldots, C_{n}\right]$. Thus, we have $\left\{C_{0}, \ldots, C_{n}\right\} \in \mathcal{C}^{0}(\mathcal{L})$. With this, we obtain

$$
\sigma_{n+k}^{0}(\mathcal{L})+\epsilon>\sum_{i=0}^{n+k} \frac{1}{1+\Lambda\left(C_{i}\right)} \geq \sigma^{0}(\mathcal{L})+\frac{k}{1+\max _{\mathcal{L}^{0}} \Lambda} .
$$

Letting $\epsilon \rightarrow 0$, the claim folows.

Returning to the main line, let $m=1$ and $k=m-1$ in (34). We obtain

$$
\sigma_{m}^{0}(\mathcal{L}) \leq \sigma_{1}^{0}(\mathcal{L})+\frac{m-1}{1+\max _{\mathcal{L}^{0}} \Lambda} \leq 1+\frac{m-1}{2}=\frac{m+1}{2} .
$$

The upper bound for $\sigma_{m}^{0}(\mathcal{L})$ follows. If $\sigma_{m}^{0}(\mathcal{L})=(m+1) / 2$, then $\max _{\mathcal{L}^{0}} \Lambda=$ $\max _{\partial \mathcal{L}} \Lambda=1$. Thus, $\Lambda=1$ and $\mathcal{L}$ is symmetric. The proposition follows.

\section{LOCAL MAXima of THE Distortion}

We first study the distance function $\Delta: \partial \mathcal{L} \rightarrow \mathbf{R}$, defined by

$$
\Delta(C)=d(C, \mathcal{O}), \quad C \in \partial \mathcal{L} .
$$

Let $C \in \partial \mathcal{L}$. We define the derivative $D_{\tau} \Delta(C)$ with respect to an oriented 2dimensional affine subspace $\tau \subset \mathcal{E}$ that contains $\mathcal{O}$ and $C$. We parametrize the boundary curve $\partial \mathcal{L} \cap \tau$ by the angular variable measured from $C$ in the positive direction so that for $C(t) \in \partial \mathcal{L} \cap \tau$, we have $t=\angle C \mathcal{O} C(t)$. It follows from convexity that $t \mapsto C(t)$ has one-sided derivatives. We introduce the angle

$$
\alpha=\alpha_{\tau}(C)=\angle \mathcal{O} C C^{\prime}, \quad C^{\prime}=\lim _{t \rightarrow 0^{+}} \frac{C(t)-C}{t} .
$$


For any function $f: \partial \mathcal{L} \rightarrow \mathbf{R}$ differentiable on $\partial \mathcal{L} \subset \mathcal{E}$, we define

$$
D_{\tau} f(C)=\lim _{t \rightarrow 0^{+}} \frac{f(C(t))-f(C)}{t} .
$$

We now calculate $D_{\tau} \Delta$. (Note that $\Delta$ is differentiable, as $\mathcal{O}$ is away from $\partial \mathcal{L}$.)

Lemma. We have

$$
D_{\tau} \Delta(C)=-\Delta(C) \cot \alpha .
$$

Proof. Choose a coordinate system on $\tau$ such that $\mathcal{O}$ is the origin and $C$ is on the positive first axis. With respect to this coordinate system, we have $C(t)=$ $(\Delta(C(t)) \cos t, \Delta(C(t)) \sin t)$. Differentating, (35) follows from the formula

$$
\begin{aligned}
\lim _{t \rightarrow 0^{+}} \frac{C(t)-C}{t} & =\left(\Delta(C), \lim _{t \rightarrow 0^{+}} \frac{\Delta(C(t))-\Delta(C)}{t}\right) \\
& =\left(\Delta(C), D_{\tau} \Delta(C)\right) .
\end{aligned}
$$

By (35), we have

$$
D_{\tau} \log \Lambda(C)=\cot \alpha^{o}-\cot \alpha,
$$

where $\alpha=\alpha_{\tau}(C)$ and $\alpha^{o}=\alpha_{\tau}\left(C^{o}\right)$.

Corollary 1. Let $C \in \partial \mathcal{L}$. We have

$$
D_{\tau} \log \Lambda(C) \leq 0 \quad \text { iff } \quad \alpha \leq \alpha^{o} .
$$

Let $\tau^{\prime}$ be $\tau$ oppositely oriented and $\alpha^{\prime}=\alpha_{\tau^{\prime}}(C)$. By convexity, $\alpha+\alpha^{\prime} \leq \pi$ and equality holds iff $C$ is a smooth point of $\partial \mathcal{L} \cap \tau$ [1]. $C$ is a smooth point of $\partial \mathcal{L}$ iff $\alpha+\alpha^{\prime}=\pi$ for all $\tau \supset[\mathcal{O}, C]$. (For example, if $\mathcal{L}$ is a polytope, then $\alpha+\alpha^{\prime}=\pi$ if $C$ is on a side of the polygon $\partial \mathcal{L} \cap \tau$, and $\alpha+\alpha^{\prime}<\pi$ if $C$ is a vertex of $\partial \mathcal{L} \cap \tau$.)

Corollary 2. Assume $D_{\tau} \log \Lambda(C) \leq 0$ for all $\tau \supset[\mathcal{O}, C]$. If $C$ is a smooth point of $\partial \mathcal{L} \cap \tau$, then so is $C^{o}$. In addition, the tangent lines to $\partial \mathcal{L} \cap \tau$ at $C$ and at $C^{o}$ are parallel.

Proof. By Corollary 1, we have $\alpha \leq \alpha^{o}$ and $\alpha^{\prime} \leq \alpha^{o}$, where $\alpha^{o}=\alpha_{\tau}\left(C^{o}\right)$ and $\alpha^{\prime o}=\alpha_{\tau^{\prime}}\left(C^{o}\right)$. Adding, we have

$$
\alpha+\alpha^{\prime} \leq \alpha^{o}+\alpha^{\prime 0} \leq \pi .
$$

Since $C$ is a smooth point, $\alpha+\alpha^{\prime}=\pi$. Thus $\alpha=\alpha^{o}$ and $\alpha^{\prime}=\alpha^{\prime o}$. The corollary follows.

Let $C \in \partial \mathcal{L}$. Consider the set of all affine subspaces $\mathcal{A} \subset \mathcal{E}$ such that $C$ is in the interior of $\partial \mathcal{L} \cap \mathcal{A}$ (relative to $\mathcal{A}$ ). This set has a maximal element $\mathcal{A}_{C}$. (By convexity, if $\mathcal{A}$ and $\mathcal{A}^{\prime}$ are elements of this set, then so is their affine span.)

We call $C$ a $k$-flat point, where $k=\operatorname{dim} \mathcal{A}_{C}$. By definition, 0 -flat points are the extremal points of $\mathcal{L}$. If $C$ is $k$-flat, $k>0$, then the boundary of $\partial \mathcal{L} \cap \mathcal{A}_{C}$ (relative to $\mathcal{A}_{C}$ ) consists of $l$-flat points with $l<k$. (If $C^{\prime} \in \partial \mathcal{L} \cap \mathcal{A}_{C}$, then $\mathcal{A}_{C^{\prime}} \subset \mathcal{A}_{C}$.)

Let $\mathcal{A} \subset \mathcal{E}$ be an affine subspace. We denote by $\mathcal{A}_{0} \subset \mathcal{E}$ the linear subspace obtained from $\mathcal{A}$ by translation. Given affine subspaces $\mathcal{A}$ and $\mathcal{A}^{\prime}$, we say that $\mathcal{A}$ is parallel to $\mathcal{A}^{\prime}$ if $\mathcal{A}_{0} \subset \mathcal{A}_{0}^{\prime}$. Note that this relation is not symmetric unless $\operatorname{dim} \mathcal{A}=\operatorname{dim} \mathcal{A}^{\prime}$.

Proposition. Let $C \in \partial \mathcal{L}$ be a $k$-flat point, $k>0$, and assume that $\Lambda$ has a local maximum at $C$. Then $C^{o}$ is l-flat with $l \geq k$ and $\mathcal{A}_{C}$ is parallel to $\mathcal{A}_{C^{o}}$. 
Proof. Let $\tau \supset[\mathcal{O}, C]$ be an affine plane such that $\tau \cap \mathcal{A}_{C}$ is nontrivial. Then $\alpha+\alpha^{\prime}=\pi$, and $C$ is a smooth point of $\partial \mathcal{L} \cap \tau$. By Corollary 2, $C^{o}$ is also a smooth point, and the tangent lines to $\partial \mathcal{L} \cap \tau$ at $C$ and at $C^{o}$ are parallel. We claim that $\tau$ intersects $\mathcal{A}_{C^{o}}$ nontrivially. Let $C^{\prime} \in \partial \mathcal{L} \cap \tau \cap \mathcal{A}_{C}$. Let $C^{\prime \prime}$ be the intersection of the line passing through $C$ and $\mathcal{O}$ with the tangent line to $\partial \mathcal{L} \cap \tau$ at $C^{o}$. By convexity, we have $C^{\prime o} \in\left[\mathcal{O}, C^{\prime \prime}\right]$. Hence

$$
\Lambda\left(C^{\prime}\right)=\frac{\Delta\left(C^{\prime}\right)}{\Delta\left(C^{\prime o}\right)} \geq \frac{\Delta\left(C^{\prime}\right)}{\Delta\left(C^{\prime \prime}\right)}=\Lambda(C)
$$

where the last equality is because the tangent lines are parallel. Since $\Lambda$ has a local maximum at $C$, equality holds provided $C^{\prime}$ is close to $C$. This means that $C^{\prime \prime}=C^{\prime o}$. Varying $C^{\prime}$ (close to $C$ ) we obtain that $\partial \mathcal{L} \cap \tau$ contains an open neighborhood of $C^{o}$ in the tangent line at $C^{o}$. This means that $\tau \cap \mathcal{A}_{C^{o}}$ is nontrivial, or equivalently, that the tangent line to $\partial \mathcal{L} \cap \tau$ at $C^{o}$ is contained in $\mathcal{A}_{C}$. The proposition follows.

Corollary. We have

$$
\max _{\partial \mathcal{L}} \Lambda=\max _{\mathcal{L}^{0}} \Lambda
$$

Proof. If $\Lambda$ attains its maximum at a nonextremal point $C \in \partial \mathcal{L}$, then $C$ is $k$-flat for some $k>0$. By the Krein-Milman theorem, $\partial \mathcal{L} \cap \mathcal{A}_{C}$ contains an extremal point $C^{\prime}$. Since $\mathcal{A}_{C}$ is parallel to $\mathcal{A}_{C^{o}}$, we have $\Lambda\left(C^{\prime}\right)=\Lambda(C)$.

Example. Let $S O(4)=S U(2) \cdot S U(2)^{\prime}$ be the natural product structure, and let $\mathcal{L}=\left(\mathcal{L}_{3}^{2}\right)^{S U(2)^{\prime}}$ be the moduli of quadratic eigenmaps $f: S^{3} \rightarrow S^{N}$ that are $S U(2)$ equivariant [4. Then the Hopf map $f: S^{3} \rightarrow S^{2}$ corresponds to an extremal point $C$ of $\mathcal{L}$ with maximal distortion $(=3 \sqrt{2})$. Its antipodal, the complex Veronese map $f^{o}: S^{3} \rightarrow S^{5}$, corresponds to a 2 -flat point $C^{o}$. This shows that in the Proposition above $l>k$ does occur.

\section{Proof of Theorem F}

Assume that there exists a minimal $m$-configuration $\left\{C_{0}, \ldots, C_{m}\right\}$ such that $\mathcal{O}$ is in the relative boundary of the polytope $\left[C_{0}, \ldots, C_{m}\right]$ in the affine span of $\left\{C_{0}, \ldots, C_{m}\right\}$. Then, renumbering the points if necessary, $\mathcal{O} \in\left[C_{0}, \ldots, C_{m_{0}}\right]$ for some $m_{0}<m$. Clearly, $\left\{C_{0}, \ldots, C_{m_{0}}\right\}$ is minimal for $\sigma_{m_{0}}(\mathcal{L})$, that is,

$$
\sigma_{m_{0}}(\mathcal{L})=\sum_{i=0}^{m_{0}} \frac{1}{1+\Lambda\left(C_{i}\right)}
$$

Moreover, for $m_{0}<i \leq m, \Lambda$ must attain its maximum at $C_{i}$, since $C_{i}$ is a point in a minimal configuration and it has no role in the condition $\mathcal{O} \in\left[C_{0}, \ldots, C_{m}\right]$. We obtain

$$
\Lambda\left(C_{m_{0}+1}\right)=\ldots=\Lambda\left(C_{m}\right)=\max _{\partial \mathcal{L}} \Lambda
$$

Using this and (37), we have

$$
\sigma_{m}(\mathcal{L})=\sum_{i=0}^{m} \frac{1}{1+\Lambda\left(C_{i}\right)}=\sigma_{m_{0}}(\mathcal{L})+\frac{m-m_{0}}{1+\max _{\partial \mathcal{L}} \Lambda} .
$$

In particular, we obtain (13).

From now on we can assume that for any minimal $m$-configuration $\left\{C_{0}, \ldots, C_{m}\right\}$, $\left[C_{0}, \ldots, C_{m}\right]$ is an $m$-simplex with $\mathcal{O}$ in its interior. The latter condition implies that $\Lambda$ has a local maximum at each $C_{i}$. 
Assume that $C_{i}$ is not an extremal point. By the proposition in the previous section, the nontrivial $\mathcal{A}_{C_{i}}$ is parallel to $\mathcal{A}_{C_{i}^{o}}$. We can move $C_{i}$ to an extremal point in the boundary of $\partial \mathcal{L} \cap \mathcal{A}_{C_{i}}$ without altering $\Lambda\left(C_{i}\right)$ and also maintaining the condition $\mathcal{O} \in\left[C_{0}, \ldots, C_{m}\right]$. Theorem F follows.

\section{REFERENCES}

[1] Berger M., Geometry I-II, Springer, 1994. MR1295239 (95g:51001)

[2] Grünbaum B., Convex Polytopes, Springer, 2003. MR1976856 (2004b:52001)

[3] Moore, J.D., Isometric immersions of space forms into space forms, Pacific J. Math. 40 (1972) 157-166. MR0305312 (46:4442)

[4] Toth G., Simplicial Intersections of a Convex Set and Moduli for Spherical Minimal Immersions, Michigan Math. J. 52 (2004) 341-359. MR2069804(2005e:53097)

[5] Toth G., Finite Möbius Groups, Minimal Immersions of Spheres, and Moduli, Springer, 2002. MR:1863996 (2002i:53082)

[6] Toth G., Infinitesimal rotations of isometric minimal immersions between spheres, Amer. J. Math. 122 (2000) 117-152. MR1737259 (2000j:53085)

[7] Toth G.,Universal constraints on the range of eigenmaps and spherical minimal immersions, Trans. Amer. Math. Soc. 351, No. 4 (1999) 1423-1443. MR1487632 (99f:53067)

Department of Mathematics, Rutgers University, Camden, New Jersey 08102

E-mail address: gtoth@crab.rutgers.edu 